\title{
Modeling of coherent ultrafast magneto-optical experiments: Light-induced molecular mean-field model
}

Y. Hinschberger, and P.-A. Hervieux

Citation: Journal of Applied Physics 118, 243902 (2015);

View online: https://doi.org/10.1063/1.4938387

View Table of Contents: http://aip.scitation.org/toc/jap/118/24

Published by the American Institute of Physics

\section{Articles you may be interested in}

Perspective: Ultrafast magnetism and $\mathrm{THz}$ spintronics

Journal of Applied Physics 120, 140901 (2016); 10.1063/1.4958846

The influence of the excitation pulse length on ultrafast magnetization dynamics in nickel

Structural Dynamics 2, 024501 (2015); 10.1063/1.4914891

Spin dynamics of the antiferromagnetic-to-ferromagnetic phase transition in FeRh on a sub-picosecond time scale

Applied Physics Letters 85, 2857 (2004); 10.1063/1.1799244

Non-thermal optical excitation of terahertz-spin precession in a magneto-optical insulator

Applied Physics Letters 108, 032404 (2016); 10.1063/1.4940241

Ultrafast demagnetization by hot electrons: Diffusion or super-diffusion?

Structural Dynamics 3, 055101 (2016); 10.1063/1.4964892

\section{Scilight}

Sharp, quick summaries illuminating the latest physics research

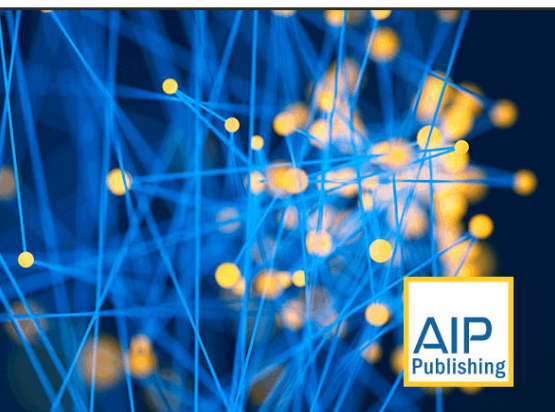




\title{
Modeling of coherent ultrafast magneto-optical experiments: Light-induced molecular mean-field model
}

\author{
Y. Hinschberger ${ }^{1}$ and P.-A. Hervieux ${ }^{2}$ \\ ${ }_{1}^{1}$ Instituto de Física dos Materiais da Universidade do Porto, Departamento de Física et Astronomia, \\ Rua do campo Alegre, 687, 4169-007 Porto, Portugal \\ ${ }^{2}$ Institut de Physique et Chimie des Matériaux de Strasbourg, Université de Strasbourg, \\ CNRS UMR 7504 BP 43 - F-67034 Strasbourg Cedex 2, France
}

(Received 11 September 2015; accepted 9 December 2015; published online 24 December 2015)

\begin{abstract}
We present calculations which aim to describe coherent ultrafast magneto-optical effects observed in time-resolved pump-probe experiments. Our approach is based on a nonlinear semi-classical Drude-Voigt model and is used to interpret experiments performed on nickel ferromagnetic thin film. Within this framework, a phenomenological light-induced coherent molecular mean-field depending on the polarizations of the pump and probe pulses is proposed whose microscopic origin is related to a spin-orbit coupling involving the electron spins of the material sample and the electric field of the laser pulses. Theoretical predictions are compared to available experimental data. The model successfully reproduces the observed experimental trends and gives meaningful insight into the understanding of magneto-optical rotation behavior in the ultrafast regime. Theoretical predictions for further experimental studies are also proposed. (C) 2015 AIP Publishing LLC.

[http://dx.doi.org/10.1063/1.4938387]
\end{abstract}

\section{INTRODUCTION}

Within the large and active domain of femtomagnetism, ultrafast coherent magnetism is a very challenging problem, which has been intensively explored over the last two decades. ${ }^{1-3}$ The ultrafast demagnetization induced by a femtosecond laser pulse on ferromagnetic films occurs via multiple thermal and non-thermal processes involving spins, electrons, photons, and phonons. There is now almost a consensus that, as soon as the system is thermalized, the magnetization dynamics can be attributed to the interaction between spins and phonons (but not in non-thermalized situations, for instance, near the Curie point ${ }^{4,5}$ ). However, the origin of ultrafast demagnetization in the first femtoseconds remains unclear. ${ }^{6-14}$ The understanding of such a phenomenon is experimentally very difficult to access due to the thermal effects (deposited by the laser pulse) and dissipated on longer time-scales.

Recently, using a clever idea, Bigot et al. have been able to eliminate the incoherent demagnetization part associated with the spin thermalization and have measured a coherent magneto-optical signal reflecting a pure magnetic response of the nickel film occurring during the laser propagation. ${ }^{9}$ Within the framework of the current debate on the mechanisms at the origin of ultrafast demagnetization, this work constitutes an important advance. Moreover, due to the various sources of magnetic ordering in nanostructures, this result opens the way for further exciting experimental and theoretical investigations. Let us mention a few of them: (i) Will the result be different for antiferromagnetic systems or diluted magnetic semiconductors? (ii) Could the exchange interaction be coherently modified by the laser electromagnetic field? (iii) Does the relativistic spin-photon interaction, proposed in Ref. 9, play an important role? (iv) Can the coherent spin and orbital dynamics be separated as they are in experiments performed on longer time-scales? ${ }^{15}$

Before tackling these interesting issues, it is important to understand what exactly is measured in such an experiment. In the present work, we analyze the data presented in Ref. 9 and model this experiment in the general framework of anisotropic and nonlinear optics. To this end, we propose using the nonlinear Drude-Voigt model. Despite being far from a sophisticated first-principles approach, ${ }^{16}$ this model has produced encouraging results underscoring the nonlinear role of charge carriers in the magneto-optical rotation. ${ }^{17}$ To circumvent the problem of employing microscopic mechanisms, the modification of the magnetic state created by the laser field is explained using of a phenomenological light-induced molecular mean-field. Its analytical expression is postulated with the help of considerations based on the relativistic spin-orbit coupling (SOC) between spins and photons. This quite simple approach gives meaningful information on the relative importance of the charge and spin contributions to the coherent magneto-optical rotation.

In Section II, we remind some basics about the distinction between coherent and incoherent ultrafast magneto-optical manipulations and we analyze the experiment performed in Ref. 9. A theoretical model is presented in Section III. Then, in Section IV, theoretical predictions of the magnetooptical rotation angles are compared to the experimental data of Ref. 9, and some proposals on further feasible experiments are given. Finally, in Section V, limitations and improvements of the model are discussed and some conclusions are given.

\section{COHERENT ULTRAFAST MAGNETO-OPTICS}

In the general context of femtomagnetism, we here remind the principle of the experiment described in Ref. 9. 
The magnetization dynamics is experimentally determined using the time-dependent magneto-optical Kerr effect (MOKE). After a strong excitation induced by a pump laser pulse, the magnetization of the material sample is probed by measuring the rotation angle of the outgoing probe pulse with a time delay $\tau$ (reflected (transmitted) beam in the Kerr (Faraday) geometry). It is usually admitted that the magnetooptical rotation is proportional to the magnetization state of the material sample. As a general trend, after the interaction of the femtosecond laser pulse and the magnetic sample, ultrafast demagnetization curves usually exhibit a quick decrease in the first hundred femtoseconds, which is followed by a relaxation process. Later, a new equilibrium state is reached on longer timescales where oscillations may be observed depending on the internal parameters governing the effective magnetic field. From a theoretical point of view, the usual demagnetization curves are well described using the Landau-Lifschitz-Gilbert equation (LLG), which is a precessional damped equation with a stochastic Langevin term. The latter depends on the temperature via a two-temperature or three-temperature model ${ }^{18}$ and mimics the perturbation due to the thermal effects. This has become a full-fledged art, and sophisticated open source softwares are now available to model such effects. ${ }^{19}$ However, what happens in the first femtoseconds is still a matter of experimental and theoretical debate.

In this context, an interesting question arises: how to distinguish the part of the demagnetization rate originating from the heat deposited by the laser pulse from the one induced by the electromagnetic field? In the present investigation, the latter is named "coherent" and is associated with the electromagnetic response induced by the external electromagnetic perturbation. To understand the coherent ultrafast magneto-optical phenomena occurring during the pulse propagation, Bigot et al. performed magneto-optical measurements on nickel and $\mathrm{CoPt}_{3}$ ferromagnetic films where the incoherent magnetic effects due to the heat were eliminated. Figure 1 is a sketch of the setup of this experiment where results are presented for a nickel film. The 48 -fs pulses were centered at $799 \mathrm{~nm}$ and the absorbed energy filled by the pump was $0.4 \mathrm{~mJ} / \mathrm{cm}^{2}$. The main idea is that the incoherent part of the magneto-optical rotation is not sensitive to the polarization state of the probe or pump pulses. Hence, using a pump-probe set-up (sketched in Fig. 1(a)) they performed magneto-optical measurements in two different configurations: the first one where pump and probe polarizations were parallel $\left(\theta_{\mathrm{PP}}=0^{\circ}\right)$ and the second where the latter were perpendicular $\left(\theta_{\mathrm{PP}}=90^{\circ}\right)$. In both configurations with beams propagating along an $\mathbf{e}_{z}$ axis, the probe polarization was kept identical $\mathbf{E}^{\text {probe }}=E^{\text {probe }} \mathbf{e}_{x}$, whereas the pump polarization has been modified ( $\mathbf{E}^{\text {pump }}=E^{\text {pump }} \mathbf{e}_{x}$ in the parallel configuration and $\mathbf{E}^{\text {pump }}=E^{\text {pump }} \mathbf{e}_{y}$ in the perpendicular configuration). The normalized rotation angle $\Delta \theta / \theta$ in both cases is shown in Fig. 1(b). Let us emphasize that $\Delta \theta / \theta$ is defined as $\frac{\Delta \theta(\tau)}{\theta}=\frac{\theta_{\text {probe }}^{\text {pump }}(\tau)-\theta_{\text {alone }}^{\text {probe }}}{\theta_{\text {alone }}^{\text {probe }}}$, where $\theta_{\text {probe }}^{\text {pump }}(\tau)$ stands for the rotation angle of the reflected probe beam in the presence of the pump for different delays $\tau$ and $\theta_{\text {alone }}^{\text {probe }}$ is the static rotation of the probe without the pump perturbation. In both cases, the quantities $\Delta \theta / \theta$ are determined with $\Delta \theta / \theta=\frac{1}{2}\left[(\Delta \theta / \theta)_{+H}-(\Delta \theta / \theta)_{-H}\right]$, where $H$ is the applied magnetic field polarizing the sample.

One can see in Fig. 1(b) that the general trend of a demagnetization signal is observed, with however a difference between the two configurations of around $\tau= \pm 100 \mathrm{fs}$ where a non-negligible shift is observed. The two signals remain identical after $200 \mathrm{fs}$ when the system is thermalized. This difference is evaluated by subtracting the Kerr rotations
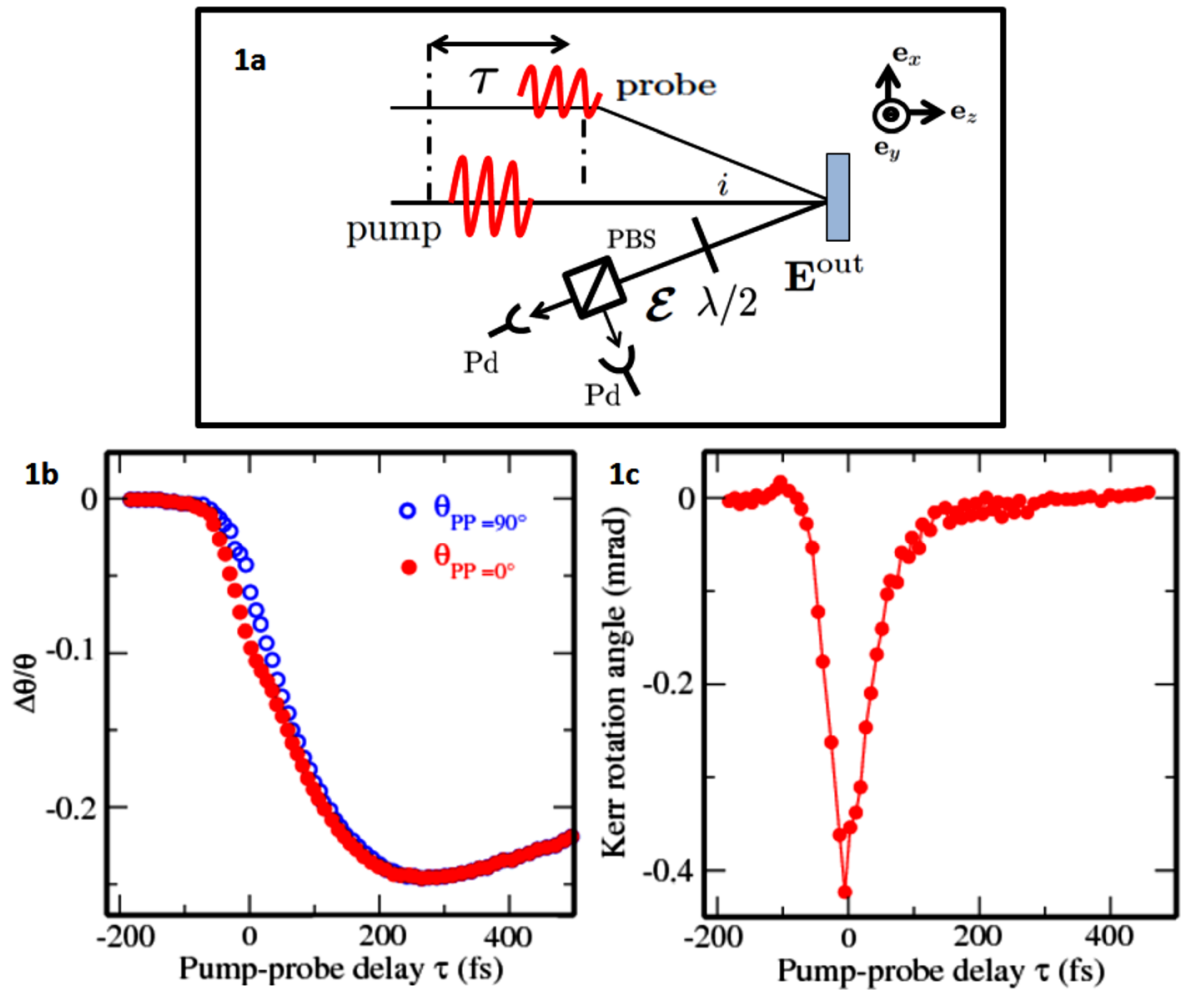

FIG. 1. (a) Sketch of the experimental setup of Ref. 9. (b) Normalized Kerr rotation angle for nickel in parallel and perpendicular configurations. (c) Coherent magnetic signal. Open and full circles represent the experimental data taken from Ref. 9. 
$\left(\theta_{\text {probe }}^{\text {pump }}\right)_{\mathrm{PP}=0^{\circ}}-\left(\theta_{\text {probe }}^{\text {pump }}\right)_{\mathrm{PP}=90^{\circ}}$ as a function of the time delay $\tau$ and is presented in Fig. 1(c). The subtraction cancels out the incoherent demagnetization part, which is identical in the two configurations. The signal which is obtained, called a coherent magnetic signal, is sensitive to the pump electromagnetic perturbation. This result shows that the magnetic state can be coherently modified by a laser pulse on a femtosecond time-scale. More importantly, its magnitude in terms of the Kerr rotation angle is more than few tenths of milliradians because the exhibited signal illustrates a differential measurement between the $\mathbf{e}_{x}$ and $\mathbf{e}_{y}$ pump polarizations.

In their article, the authors of Ref. 9 have proposed a microscopic interpretation based on a spin-photon relativistic coupling $(e>0)$

$$
{ }^{\sigma} H=\frac{e \hbar}{2 m_{e}} \boldsymbol{\sigma} \cdot\left(\mathbf{B}+\frac{1}{2 m_{e} c^{2}} \mathbf{E} \wedge(\mathbf{p}+e \mathbf{A})\right)+\vartheta\left(m_{e}^{-3}\right),
$$

where the first and second term of Eq. (1) are, respectively, the Zeeman interaction and the SOC. By comparing magneto-optical measurements performed in the parallel $\left(\theta_{\mathrm{PP}}=0^{\circ}\right)$ and perpendicular $\left(\theta_{\mathrm{PP}}=90^{\circ}\right)$ configurations, Bigot et al. have revealed the role played by electric field polarization on the ultrafast spin dynamics occurring during the first few femtoseconds and have attributed this phenomenon to a coherent spin-photon coupling. This assumption was also supported by a complementary single-pulse-like Faraday experiment (not presented here) by following Hulme's, ${ }^{20}$ Argyres's, ${ }^{21}$ and Benneth's ${ }^{22}$ descriptions of the Faraday effect in ferromagnetic materials, where the necessary spin-orbit interaction between the electron spins and the ionic electric field can be extended to the one carried by the laser field. This interpretation has not been confirmed or refuted yet. Nevertheless, let us mention some recent work on that topic concerning: (i) the influence of a laserinduced SOC on the magneto-optical rotation of hydrogenlike systems; ${ }^{23}$ (ii) the coupling between the electron spin with an external time-dependent electromagnetic field in the framework of the Dirac-Breit equation and the Foldy Wouthuysen transformation, ${ }^{24,25}$ or the incorporation of relativistic effects in a self-consistent mean-field approach. ${ }^{26,27}$ Let us also mention a recent work ${ }^{28}$ based on $a b$-initio calculations and concluding that relativistic effects play a negligible role in the MOKE linear response. However, in the present work, we investigate the nonlinear response of ferromagnetic samples, which strongly differ from linear regime responses. From an experimental point of view, such a coherent nonlinear regime can be probed by using a femtosecond magneto-optical four-wave-mixing configuration performed on Garnet films. ${ }^{29}$ Further experiments using sub-10 femtosecond pulses, based on a novel dual-color scheme, will soon permit us to investigate coherent ultrafast magnetization dynamics. ${ }^{30}$ The understanding of the above seminal work ${ }^{9}$ is therefore of crucial interest for future works. In the present investigation, we suggest a first approach using basic concepts of anisotropic and nonlinear optics. In what follows, the incoherent effects due to the heat (temperature) will be neglected since they are experimentally eliminated.

\section{MODEL}

In this three part section, we propose to model the experiment of Ref. 9 presented in Sec. II. In Section III A, we explain how we calculate the magneto-optical rotation and we propose an analytical form for the dielectric polarization. Section III B is devoted to the determination of the dielectric tensor elements within a nonlinear Drude-Voigt model. Finally, in Section III C, we underline the importance of introducing in the modeling, a light-induced coherent molecular mean-field dependent on the pulse polarization.

\section{A. Magneto-optical rotation angle and dielectric polarization}

The magneto-optical rotation is usually determined experimentally using the device sketched in Fig. 1(a). The reflected outgoing probe field, $\mathbf{E}^{\text {out }}$, crosses a half-wave plate and is transformed into the field, $\mathcal{E}$, which is divided into its $x$ and $y$ components using a Polarizer Beam Splitter (PBS). Their intensities $\mathcal{I}_{x}$ and $\mathcal{I}_{y}$ are measured using a system of photodiodes $(\mathrm{Pd})$ in order to estimate the rotation angle defined as $\theta \approx\left(\mathcal{I}_{y}-\mathcal{I}_{x}\right) /\left(\mathcal{I}_{y}+\mathcal{I}_{x}\right)$. Such a modeling of the rotation was performed in Ref. 31 , and experimental details concerning the procedure can be found in Refs. 32 and 33.

In what follows, we assume that the angle between the pump and the probe pulses is negligible (i.e., the angle $i$ in Fig. 1(a) can be taken as $i \approx 0$ ) and therefore, the electric fields propagate along the $\mathbf{e}_{z}$ axis with components in the $\left(\mathbf{e}_{x}, \mathbf{e}_{y}\right)$ plane, which is parallel to the plane of the material sample. Therefore, the components of the outgoing field, $\mathbf{E}^{\text {out }}$, are $\left(E_{x}^{\text {out }} \mathbf{e}_{x}, E_{y}^{\text {out }} \mathbf{e}_{y}\right)$.

According to the authors of Ref. 9, the half-wave plate is tilted at an angle $\pi / 8$ with respect to the laboratory frame and using its Jones's matrix $\left[M_{\lambda}\right]$, the field $\mathcal{E}$ reaching the PBS may be written as

$$
\mathcal{E}=\left[M_{\lambda}\right] \mathbf{E}^{\text {out }}=\left[\begin{array}{cc}
1 & 1 \\
1 & -1
\end{array}\right]\left|\begin{array}{l}
E_{x}^{\text {out }} \\
E_{y}^{\text {out }}
\end{array}=\right| \begin{aligned}
& E_{x}^{\text {out }}+E_{y}^{\text {out }} \\
& E_{x}^{\text {out }}-E_{y}^{\text {out }}
\end{aligned}
$$

The associated intensities are defined as $I_{k}=\frac{1}{T_{d}} \int_{0}^{T_{d}} d t\left(\mathcal{E}_{k}^{*} \mathcal{E}_{k}\right)$ for $(k=x, y)$, where $T_{d}$ is the photodiode integration time and $\mathcal{E}_{k}^{*}$ is the complex conjugate of $\mathcal{E}_{k}$. However, from a theoretical point of view, it is sufficient to calculate $I_{k}$ as $I_{k}=\int_{-\infty}^{\infty} d t\left(\mathcal{E}_{k}^{*} \mathcal{E}_{k}\right)$ since the magneto-optical rotation angle $\theta$ is determined with the following normalized quantity:

$$
\theta=\frac{\mathcal{I}_{y}-\mathcal{I}_{x}}{\mathcal{I}_{y}+\mathcal{I}_{x}}=\frac{\int d t\left(\mathcal{E}_{y}^{*} \mathcal{E}_{y}\right)-\int d t\left(\mathcal{E}_{x}^{*} \mathcal{E}_{x}\right)}{\int d t\left(\mathcal{E}_{y}^{*} \mathcal{E}_{y}\right)+\int d t\left(\mathcal{E}_{x}^{*} \mathcal{E}_{x}\right)}
$$

Then, one has to determine the expression of the outgoing field. Here, we assume that the components of the reflected electric field are proportional to the ones of the sample dielectric polarization function induced by the pump and the probe pulses. This leads to $\mathbf{E}^{\text {out }}=\left(E_{x}^{\text {out }}, E_{y}^{\text {out }}\right) \equiv\left(P_{x},-P_{y}\right)$, where $\mathbf{P}$ is the dielectric polarization.

We consider that the electric field of the pump may be written as: $\mathbf{E}^{\text {pump }}(t) \approx \mathbf{E}_{0}^{\text {pump }} e^{-\frac{t^{2}}{2 \Gamma^{2}}}\left(e^{i \Omega t}+e^{-i \Omega t}\right)$, where $\Omega$ is 
the central pulsation, $\Gamma$ is associated with the pulse duration, and the amplitude of the electric field is $\mathbf{E}_{0}^{\text {pump }}$ $=\left(E_{0 x}^{\text {pump }} \mathbf{e}_{x}, E_{0 y}^{\text {pump }} \mathbf{e}_{y}\right)$. Similarly, the probe electric field delayed by a time $\tau$ may be written as: $\mathbf{E}^{\text {probe }}(t, \tau)$ $\approx \mathbf{E}_{0}^{\text {probe }} e^{-\frac{(t-\tau)^{2}}{2 \gamma^{2}}}\left(e^{i \omega(t-\tau)}+e^{-i \omega(t-\tau)}\right)$ with the parameters $\omega, \gamma$, and $\mathbf{E}_{0}^{\text {probe }}=\left(E_{0 x}^{\text {probe }} \mathbf{e}_{x}, E_{0 y}^{\text {probe }} \mathbf{e}_{y}\right)$.

Hence, by working in the Fourier space for a damped $\omega$ mode $\mathbf{E} \equiv \mathbf{E}_{0} e^{-\frac{t^{2}}{2 \gamma^{2}}+i \omega t}$, and within the framework of a nonlinear Drude-Voigt model (see Section IIIB), the complex polarization vector of the system may be expressed as

$$
\begin{aligned}
\mathbf{P}_{\omega}(t, \tau)= & \epsilon_{0}[\chi(\omega, \gamma)] \mathbf{E}^{\text {probe }}(t, \tau)+\epsilon_{0}\left[\epsilon^{(3)}(\omega, \gamma)\right] e^{-\frac{(t-\tau)^{2}}{\gamma^{2}}} \\
& \times\left\|\mathbf{E}_{0}^{\text {probe }}\right\|^{2} \mathbf{E}^{\text {probe }}(t, \tau)+\epsilon_{0}\left[\epsilon_{\text {probe }}^{\text {pump }}(\omega, \Omega, \gamma, \Gamma)\right] e^{-\frac{t^{2}}{\Gamma^{2}}} \\
& \times\left\|\mathbf{E}_{0}^{\text {pump }}\right\|^{2} \mathbf{E}^{\text {probe }}(t, \tau),
\end{aligned}
$$

where $[\chi(\omega, \gamma)]$ and $\left[\epsilon^{(3)}(\omega, \gamma)\right]$ are, respectively, the linear dielectric susceptibility tensor and the dielectric tensor related to the nonlinear third-order polarization fields created by the probe pulse. $\left[\epsilon_{\text {probe }}^{\text {pump }}(\omega, \Omega, \gamma, \Gamma)\right]$ is the dielectric tensor caused by the nonlinear interaction created by the pump pulse and carried by the probe pulse. One may also write $[\chi(\omega, \gamma)]$ as $[\chi(\omega, \gamma)]=([\epsilon(\omega, \gamma)]-1)$, where $[\epsilon(\omega, \gamma)]$ is the dielectric tensor defined as $\mathbf{D}=\epsilon_{0}[\epsilon(\omega, \gamma)] \mathbf{E}=\left(\epsilon_{0} \mathbf{E}+\mathbf{P}\right)$ with $\mathbf{P}=\epsilon_{0}[\chi(\omega, \gamma)] \mathbf{E}$. In what follows, all the dielectric tensors $\left[\epsilon^{(k)}\right]$ are written according to the general form

$$
\left[\epsilon^{(k)}\right]=\left[\begin{array}{ccc}
\epsilon_{x x}^{(k)} & \epsilon_{x y}^{(k)} & 0 \\
-\epsilon_{x y}^{(k)} & \epsilon_{x x}^{(k)} & 0 \\
0 & 0 & X
\end{array}\right]
$$

where $\epsilon_{x x}^{(k)}$ and $\epsilon_{x y}^{(k)}$ are, respectively, the isotropic and the anisotropic elements and $X$ stands for anything.

This formalism allows us to add up the different tensors and to define the following ones: (i) the total nonlinear dielectric tensor related to the probe $\left[\epsilon^{\mathrm{nl}}(\omega, \gamma)\right]=$ $([\epsilon(\omega, \gamma)]-1)+\left[\epsilon^{(3)}(\omega, \gamma)\right] e^{-\frac{(t-\tau)^{2}}{\gamma^{2}}}\left\|\mathbf{E}_{0}^{\text {probe }}\right\|^{2}$ and (ii) the total nonlinear dielectric tensor including the pump perturbation $\left[\epsilon^{\mathrm{nlp}}(\omega, \gamma, \Omega, \Gamma)\right]=\left[\epsilon^{\mathrm{nl}}(\omega, \gamma)\right]+\left[\epsilon_{\text {probe }}^{\text {pump }}(\omega, \Omega, \gamma, \Gamma)\right] e^{-\frac{t^{2}}{\Gamma^{2}}}\left\|\mathbf{E}_{0}^{\text {pump }}\right\|^{2}$. The associated isotropic and anisotropic tensor elements may thus be written as

$$
\begin{aligned}
\epsilon_{i j}^{\mathrm{nl}} & =\left(\epsilon_{i j}-1\right)+\epsilon_{i j}^{(3)} e^{-\frac{(t-\tau)^{2}}{\gamma^{2}}}\left(\left(E_{0 x}^{\text {probe }}\right)^{2}+\left(E_{0 y}^{\text {probe }}\right)^{2}\right) \\
\epsilon_{i j}^{\mathrm{nlp}} & =\epsilon_{i j}^{\mathrm{nl}}+\epsilon_{\text {probe } i j}^{\text {pump }} e^{-\frac{t^{2}}{\Gamma^{2}}}\left(\left(E_{0 x}^{\text {pump }}\right)^{2}+\left(E_{0 y}^{\text {pump }}\right)^{2}\right) .
\end{aligned}
$$

Therefore, using Eqs. (4) and (6), the components of the outgoing field $\mathbf{E}^{\text {out }}$ may be expressed as

$$
\mathbf{E}^{\text {out }} \equiv\left|\begin{array}{c}
P_{x} \\
-P_{y}
\end{array}=\right| \begin{aligned}
& \epsilon_{x x}^{(k)} E_{x}^{\text {probe }}+\epsilon_{x y}^{(k)} E_{y}^{\text {probe }} \\
& \epsilon_{x y}^{(k)} E_{x}^{\text {probe }}-\epsilon_{x x}^{(k)} E_{y}^{\text {probe }}
\end{aligned}
$$

where $\epsilon_{i j}^{(k)}$ denotes three different ways of irradiating the material sample: in a single-pulse configuration with (i) a weak probe pulse $\left(\epsilon_{i j}^{(k)}=\epsilon_{i j}-1\right)$, (ii) a strong probe pulse including the nonlinear regime $\left(\epsilon_{i j}^{(k)}=\epsilon_{i j}^{\mathrm{nl}}\right)$, and (iii) with the additional presence of the pump pulse $\left(\epsilon_{i j}^{(k)}=\epsilon_{i j}^{\text {nlp }}\right)$. Consequently, we are able to calculate the magneto-optical rotation in any of the three configurations: $\theta, \theta^{\text {nl }}$, and $\theta^{\text {nlp }}$.

In order to have an idea of the composition of the rotation angle $\theta^{(k)}$, let us consider a configuration where the electric field of the probe pulse is oriented along the $\mathbf{e}_{x}$ axis $\left(\mathbf{E}_{0}^{\text {probe }}=\left(E_{0 x}^{\text {probe }} \mathbf{e}_{x}, 0\right)\right)$ and the complex outgoing field may be expressed in terms of its real and imaginary parts noted, respectively, $\operatorname{Re}\left(\mathbf{E}^{\text {out }}\right)$ and $\operatorname{Im}\left(\mathbf{E}^{\text {out }}\right)$.

The $x$ and $y$ components of Eq. (7) become $\operatorname{Re}\left(\epsilon_{x x}^{(k)} E_{x}^{\text {probe }}\right)$ $+i \operatorname{Im}\left(\epsilon_{x x}^{(k)} E_{x}^{\text {probe }}\right)$ and $\operatorname{Re}\left(\epsilon_{x y}^{(k)} E_{x}^{\text {probe }}\right)+i \operatorname{Im}\left(\epsilon_{x y}^{(k)} E_{x}^{\text {probe }}\right)$, and by plugging the latter into Eq. (3), the rotation angle takes the following form:

$$
\theta^{(k)}=\frac{\int d t\left\|\mathbf{E}^{\text {probe }}(t)\right\|^{2}\left(-2 \operatorname{Re}\left(\epsilon_{x x}^{(k)}\right) \operatorname{Re}\left(\epsilon_{x y}^{(k)}\right)-2 \operatorname{Im}\left(\epsilon_{x x}^{(k)}\right) \operatorname{Im}\left(\epsilon_{x y}^{(k)}\right)\right)}{\int d t\left\|\mathbf{E}^{\text {probe }}(t)\right\|^{2}\left(\left(\operatorname{Re}\left(\epsilon_{x x}^{(k)}\right)\right)^{2}+\left(\operatorname{Re}\left(\epsilon_{x y}^{(k)}\right)\right)^{2}+\left(\operatorname{Im}\left(\epsilon_{x x}^{(k)}\right)\right)^{2}+\left(\operatorname{Im}\left(\epsilon_{x y}^{(k)}\right)\right)^{2}\right)} .
$$

The analytical expression given by Eq. (8) is useful because it indicates that the rotation angle might be more complex than a simple proportionality to the magnetization that is hidden in the anisotropic tensor elements.

Let us remember that in the present work the dielectric polarization is described with a damped $\omega$ mode $e^{-\frac{t^{2}}{2 \gamma^{2}}+i \omega t}$, which differs from a term like $e^{-\frac{t^{2}}{2 \gamma^{2}}} \cos (\omega t)$ that includes the $-\omega$ mode $e^{-\frac{t^{2}}{2 \gamma^{2}}-i \omega t}$. That gives a difference in the calculation of $\mathcal{I}_{x}$ or $\mathcal{I}_{y}$, but since the magneto-optical rotation $\theta$ is calculated with Eq. (3) (or Eq. (8)), the difference is canceled out between the numerator and denominator.

The next step is to calculate the polarization of Eq. (4). This can be achieved in various ways. We propose to make this task as simple as possible by using the nonlinear Drude-Voigt model. Despite its simplicity, this model has led to very interesting results in the modeling of the single-beam experiment described in Ref. 17. Here, the idea is to extend this model to a configuration of two delayed pulses. 


\section{B. Nonlinear Drude-Voigt model}

The following description of nickel is similar to the one that we introduced in a previous work. ${ }^{17}$ The improvements made here are twofold: (i) the incorporation of a pump-probe term and (ii) the durations of the two pulses are taken into account through the damping factors $\gamma$ and $\Gamma$. The main assumptions of the modeling are described in detail in Ref. 17:

(i) The electronic properties of nickel are described by considering bound and free electrons. We assume that the valence electronic shell can be modeled by one electron, which is screened by the nucleus and the other core electrons. One fraction $x_{b}$ of this electron is considered to be bound and another fraction $x_{f}=1-x_{b}$ is considered to be free. According to References 34 and 35 , there is 0.6 conducting electron per atomic nickel site, which fixes the value $x_{f}=0.6$. Even if nickel has a metallic character, one also has to consider the contribution of the bound electrons.

(ii) The microscopic first- and third-order displacements of the bound and free charges $\left(\mathbf{r}_{b}^{(1)}, \mathbf{r}_{f}^{(1)}, \mathbf{r}_{b}^{(3)}, \mathbf{r}_{f}^{(3)}\right)$ are determined using the anharmonic Drude-Voigt model and lead to the macroscopic polarization densities $\left(\mathbf{P}_{b}^{(1)}, \mathbf{P}_{f}^{(1)}, \mathbf{P}_{b}^{(3)}, \mathbf{P}_{f}^{(3)}\right)$ with $\mathbf{P}_{a}^{(k)}=\frac{1}{V} \sum_{i}^{N_{a}}-e \mathbf{r}_{a_{i}}^{(k)}$ where $a=b, f$ and $k=1,3$.

(iii) The total electric field acting on the charges inside the material is the sum of the external electric field of the probe beam and the polarization field generated by the charges. We assume that the dynamics of the free electrons is influenced by the bound charges dynamics, but not the contrary. Since there is no nonlinear source in the classical Drude model for free electrons, we assume that the nonlinear perturbation acting on the free electron fraction $x_{f}$ is indirectly produced through the nonlinear polarization of the bound charge carriers. We also assume that the latter originates from the anharmonic potential, which mimics the interaction with the nearest neighbors. At a microscopic level, the nonlinearity during the laser pulse mainly originates from the phase space filling and the Pauli blocking which are quantum mechanisms and therefore necessitates the use of a much more sophisticated treatment. ${ }^{36-38}$

(iv) The effective magnetic field inside the material is noted $\mathbf{B}_{\text {mat }}=B_{\text {mat }} \mathbf{e}_{z}$ and corresponds to the Weiss' molecular field.

Under these assumptions, the equation of motion for bound and free charges are given by Eqs. (9) and (10). In the Drude-Voigt model, a bound electron (of mass $m_{b}$ ) is described as a damped-harmonic oscillator (of pulsation $\omega_{0}$ and damping $\tau_{b}$ ) subject to the Lorentz force $(e>0)$. According to our assumptions, one has to add the nonlinear term $b \mathbf{r}^{3}$ originating from an anharmonic potential energy $\left(E_{p}=\frac{m_{b} \omega_{0}^{2}}{2} r^{2}-\frac{m_{b} b}{4} r^{4}\right)$ and the polarization field created by the bound charges themselves, which leads to the following equation of motion:

$$
\begin{aligned}
\ddot{\mathbf{r}}_{b}+\frac{1}{\tau_{b}} \dot{\mathbf{r}}_{b}+\omega_{0}^{2} \mathbf{r}_{b}= & -\frac{e \mathbf{E}^{\text {probe }}}{m_{b}}-\frac{e}{m_{b}} \dot{\mathbf{r}}_{b} \wedge \mathbf{B}_{\mathrm{mat}} \\
& +b \mathbf{r}_{b}^{3}-\frac{e}{m_{b} \epsilon_{0}}\left(\mathbf{P}_{b}^{(1)}+\mathbf{P}_{b}^{(3)}\right) .
\end{aligned}
$$

In the case of a free electron (of mass $m_{f}$ and damping $\left.\tau_{f}\right)$, there is no Hooke's force $\left(m \omega^{2} \mathbf{r}\right)$ in the equation, and the total electric field is the sum of the external electric field plus all the polarization fields

$$
\begin{aligned}
\ddot{\mathbf{r}}_{f}+\frac{1}{\tau_{f}} \dot{\mathbf{r}}_{f}= & -\frac{e \mathbf{E}^{\text {probe }}}{m_{f}}-\frac{e \dot{\mathbf{r}}_{f}}{m_{f}} \wedge \mathbf{B}_{\mathrm{mat}} \\
& -\frac{e}{m_{f} \epsilon_{0}}\left(\mathbf{P}_{b}^{(1)}+\mathbf{P}_{f}^{(1)}+\mathbf{P}_{b}^{(3)}+\mathbf{P}_{f}^{(3)}\right) .
\end{aligned}
$$

Starting from the above two equations, the modifications that will be incorporated are the following:

(v) We propose to work in the Fourier space with a damped $\omega$ mode. Hence, for a Gaussian electric field expressed as $\mathbf{E}^{\text {probe }} \approx \mathbf{E}_{0}^{\text {probe }} e^{-\frac{t^{2}}{2 \gamma^{2}}}\left(e^{i \omega t}+e^{-i \omega t}\right)$, the damped $\omega$ mode is $\mathbf{E}^{\text {probe }} \rightarrow \mathbf{E}_{0}^{\text {probe }} e^{-\frac{t^{2}}{2 \gamma^{2}}+i \omega t}$. This implies that the first and second time-derivative of any vector $\mathbf{r}$ read $\dot{\mathbf{r}}=\left(i \omega-\frac{t}{\gamma^{2}}\right) \mathbf{r} \quad$ and $\ddot{\mathbf{r}}=\left(-\omega^{2}-2 i \omega \frac{t}{\gamma^{2}}+\frac{t^{2}}{\gamma^{4}}-\frac{1}{\gamma^{2}}\right) \mathbf{r}$. This modification only adds a damping factor and a frequency shift of the resonance of the response functions. We note the existence of terms that depend explicitly on time $t$. However, their contributions remain smaller with respect to the ones which do not depend on time in the time domain $t \in[-100,100]$ fs. For instance, with $t=100 \mathrm{fs}, \gamma=25 \mathrm{fs}$, and $\omega=10^{15} \mathrm{rad} / \mathrm{s}$, one gets $\omega \approx$ $10 \times \frac{t}{\gamma^{2}}$ for the terms involving $\dot{\mathbf{r}}$.

(vi) The presence of the electric field of the pump pulse will induce a vector displacement $\mathbf{r}^{\text {pump }}$. Selecting the dielectric response function induced by the probe field (as is done in the experiment) does not change anything in the linear regime. However, the nonlinear term $b \mathbf{r}^{3}$ creates a pump-probe contribution (quadratic versus the pump and linear versus the probe) for the bound electrons $\mathbf{P}_{b, \text { probe }}^{(3) \text { pump }}$ and also for the free electrons $\mathbf{P}_{f \text {,probe }}^{(3) \text {,pump }}$.

(vii) As described in Section IIIC, we assume that the Weiss molecular mean-field $\mathbf{B}_{\text {mat }}$ can be modified by the external electric field and we propose an analytical expression for modeling this interaction.

In the following, we estimate the total polarization field as

$$
\begin{aligned}
\mathbf{P}= & \mathbf{P}_{b}^{(1)}+\mathbf{P}_{f}^{(1)}+\mathbf{P}_{b}^{(3)}+\mathbf{P}_{f}^{(3)} \\
& +\mathbf{P}_{b, \text { probe }}^{(3) \text { pump }}+\mathbf{P}_{f, \text { probe }}^{(3), \text { pump }}
\end{aligned}
$$

and we compare its expression with the one given in Eq. (4) in order to determine the tensor elements. In what follows, the quantity $\mathrm{E}$ denotes the damped $\omega$ mode $\mathbf{E}_{0} e^{-\frac{t^{2}}{2 \gamma^{2}}+i \omega t}$. 


\section{Dielectric part-bound electrons}

As in classical perturbation theory, ${ }^{39}$ Equation (9) is split into two parts using $\mathbf{r}_{b}(t)=\mathbf{r}_{b}^{(1)}(t)+\mathbf{r}_{b}^{(3)}(t)$

$$
\begin{gathered}
\ddot{\mathbf{r}}_{b}^{(1)}+\frac{1}{\tau_{b}} \dot{\mathbf{r}}_{b}^{(1)}+\omega_{0}^{2} \mathbf{r}_{b}^{(1)}=-\frac{e}{m_{b}} \mathbf{E}^{\text {probe }}-\frac{e \mathbf{P}_{b}^{(1)}}{m_{b} \epsilon_{0}}-\frac{e \dot{\mathbf{r}}_{b}^{(1)}}{m_{b}} \wedge \mathbf{B}_{m a t}, \\
\ddot{\mathbf{r}}_{b}^{(3)}+\frac{1}{\tau_{b}} \dot{\mathbf{r}}_{b}^{(3)}+\omega_{0}^{2} \mathbf{r}_{b}^{(3)}=b\left(\mathbf{r}_{b}^{(1)}\right)^{3}-\frac{e \mathbf{P}_{b}^{(3)}}{m_{b} \epsilon_{0}}-\frac{e \dot{\mathbf{r}}_{b}^{(3)}}{m_{b}} \wedge \mathbf{B}_{\text {mat }} .
\end{gathered}
$$

Let us first determine the linear term. By working in the Fourier space for the damped mode $\omega$ and defining the firstorder macroscopic polarization for the $N_{b}$ bound electrons, $\mathbf{P}_{b}^{(1)}=\frac{1}{V} \sum_{i}^{N_{b}}-e \mathbf{r}_{b_{i}}$, Eq. (12) is transformed into

$$
\mathbf{P}_{b}^{(1)}=\epsilon_{0} \chi_{b}^{(1)} \mathbf{E}^{\text {probe }}-i \xi_{b} \mathbf{P}_{b}^{(1)} \wedge \mathbf{e}_{z},
$$

where $\chi_{b} \equiv \chi_{b}^{(1)}(\omega, \gamma)=\frac{\mathfrak{n}_{b} e^{2}}{m_{b} \epsilon_{0} D_{b}(\omega, \gamma)}$ with $\mathfrak{n}_{\mathfrak{b}}=N_{b} / V, \xi_{b}(\omega, \gamma)$ $=\frac{e B_{\text {mat }}\left(\omega+\frac{i t}{\gamma^{2}}\right)}{m_{b} D_{b}(\omega, \gamma)} \quad$ and $\quad D_{b} \equiv D_{b}(\omega, \gamma)=\left[\left(\omega_{0}^{2}+\frac{t^{2}}{\gamma^{4}}-\frac{1}{\gamma^{2}}-\frac{t}{\tau_{b} \gamma^{2}}-\right.\right.$ $\left.\left.\frac{\mathfrak{n}_{b} e^{2}}{m_{b} \epsilon_{0}}-\omega^{2}\right)+i\left(\frac{\omega}{\tau_{b}}-\frac{2 \omega t}{\gamma^{2}}\right)\right]$ is the bound electron response function. By defining $\alpha_{b} \equiv \alpha_{b}^{(1)}(\omega, \gamma)=\frac{\chi_{b}(\omega, \gamma)}{1-\xi_{b}^{2}(\omega, \gamma)}$, Eq. (14) may be rewritten in a tensorial form as

$$
\mathbf{P}_{b}^{(1)}=\epsilon_{0} \alpha_{b}^{(1)}\left[\begin{array}{ccc}
1 & -i \xi_{b} & 0 \\
i \xi_{b} & 1 & 0 \\
0 & 0 & X
\end{array}\right] \mathbf{E}^{\text {probe }}
$$

Let us focus now on Eq. (13). It is worth mentioning that the nonlinear term $\left(\mathbf{r}_{b}^{(1)}\right)^{3}$ vibrates at pulsation $\omega$. In a single beam configuration, the latter reads $\mathbf{r}_{b}^{3}=\left(\mathbf{r}_{b}^{(1)}(\omega)+\mathbf{r}_{b}^{(1)}(-\omega)\right)^{3}$ and leads to four terms vibrating at $3 \omega, \omega,-\omega$, and $-3 \omega$. The only term which contributes to the equation is $3\left(\mathbf{r}_{b}^{(1)}(\omega)\right)^{2}$ $\mathbf{r}_{b}^{(1)}(-\omega)$ (see Ref. 17).

In the present situation, due to the presence of the pump and probe pulses, the nonlinear contribution is $\left(\mathbf{r}_{b}^{(1)}\right)^{3}=$ $\left(\mathbf{r}_{b}^{(1), \text { pump }}(\Omega)+\mathbf{r}_{b}^{(1), \text { pump }}(-\Omega)+\mathbf{r}_{b}^{(1), \text { probe }}(\omega)+\mathbf{r}_{b}^{(1), \text { probe }}(-\omega)\right)^{3}$, leading to 24 terms. However, in a classical pump-probe configuration, and in the framework of this model, the interesting terms are the ones that vibrate at the pulsation $\omega$ and are carried by the probe pulse (detailed calculation can be seen in Appendix A). Therefore, the nonlinear vector may be expressed as

$$
\begin{aligned}
\left(\mathbf{r}_{b}^{(1)}\right)^{3}= & \left(\mathbf{r}_{b}^{(1), \operatorname{pump}}(\Omega)+\mathbf{r}_{b}^{(1), \text { pump }}(-\Omega)\right. \\
& \left.+\mathbf{r}_{b}^{(1), \text { probe }}(\omega)+\mathbf{r}_{b}^{(1), \operatorname{probe}}(-\omega)\right)^{3} \\
\approx & 6\left(\mathbf{r}_{b}^{(1), \operatorname{pump}}(\Omega) \cdot \mathbf{r}_{b}^{(1), \operatorname{pump}}(-\Omega)\right) \mathbf{r}_{b}^{(1), \text { probe }}(\omega) \\
& +3\left(\mathbf{r}_{b}^{(1), \text { probe }}(\omega) \cdot \mathbf{r}_{b}^{(1), \operatorname{probe}}(\omega)\right) \mathbf{r}_{b}^{(1), \text { probe }}(-\omega),
\end{aligned}
$$

where the first term represents the nonlinear pump-probe contribution (quadratic versus the pump and linear versus the probe) and the second one is the usual nonlinear term induced by the probe. In order to calculate Eq. (16), one needs to express the displacement of a bound electron due to its interaction with the pump and the probe pulses. This can be easily performed by rewriting Eq. (12) for a bound electron interacting with any beam (pump or probe) $\mathbf{E}^{\text {beam }}$ $=\mathbf{E}_{0}^{\text {beam }} e^{-\frac{t^{2}}{2 \gamma^{\prime 2}}+i \omega^{\prime} t}$ with parameters $\omega^{\prime}, \gamma^{\prime}$

$$
\mathbf{r}_{b}^{(1), \text { beam }}\left(\omega^{\prime}, \gamma^{\prime}\right)=a^{\prime}\left(\mathbf{E}^{\text {beam }}+\frac{\mathbf{P}_{b}^{(1)}}{\epsilon_{0}}\right)-i b^{\prime} \mathbf{r} \wedge \mathbf{e}_{z},
$$

where $a^{\prime} \equiv a^{\prime}\left(\omega^{\prime}, \gamma^{\prime}\right)=-\frac{e}{m_{b} D_{b}^{\prime}\left(\omega^{\prime}, \gamma^{\prime}\right)}, b^{\prime} \equiv b^{\prime}\left(\omega^{\prime}, \gamma^{\prime}\right)=\frac{e B_{\text {mat }}\left(\omega^{\prime}+i \gamma^{\prime}\right)}{m_{b} D_{b}^{\prime}\left(\omega^{\prime}, \gamma^{\prime}\right)}$ and $D_{b}^{\prime}\left(\omega^{\prime}, \gamma^{\prime}\right)=D_{b}\left(\omega^{\prime}, \gamma^{\prime}\right)+\frac{\mathfrak{n}_{b} e^{2}}{m_{b} \epsilon_{0}}$. Then, by plugging Eq. (15) into Eq. (17), one obtains the displacement vector in a tensorial form

$$
\mathbf{r}_{b}^{(1) \text {,beam }}\left(\omega^{\prime}, \gamma^{\prime}\right)=\left[\begin{array}{ccc}
d^{\text {iso }}\left(\omega^{\prime}\right) & -i d^{\text {ani }}\left(\omega^{\prime}\right) & \\
i d^{\text {ani }}\left(\omega^{\prime}\right) & d^{\text {iso }}\left(\omega^{\prime}\right) & \\
0 & 0 & X
\end{array}\right] \mathbf{E}^{\text {beam }}
$$

where $d^{\text {iso }}\left(\omega^{\prime}\right)$ and $d^{\text {ani }}\left(\omega^{\prime}\right)$ represent, respectively, the isotropic and anisotropic displacements induced by the beam

$$
\begin{aligned}
& d^{\text {iso }}\left(\omega^{\prime}\right)=\frac{a^{\prime}}{\left(1-b^{\prime 2}\right)}\left(1+\alpha_{b}\left(1+b^{\prime} \xi_{b}\right)\right), \\
& d^{\text {ani }}\left(\omega^{\prime}\right)=\frac{a^{\prime}}{\left(1-b^{\prime 2}\right)}\left(b^{\prime}+\alpha_{b}\left(b^{\prime}+\xi_{b}\right) .\right.
\end{aligned}
$$

Let us define $f_{1}(\omega)=\left(\mathbf{r}^{(1), \operatorname{probe}}(\omega) \cdot \mathbf{r}^{(1) \text {,probe }}(\omega)\right)$ and $f_{2}(\Omega)$ $=\left(\mathbf{r}^{(1), \operatorname{pump}}(\Omega) \cdot \mathbf{r}^{(1), \operatorname{pump}}(-\Omega)\right)$ in Eq. (16). Using the expression of $\mathbf{r}_{b}^{(1) \text {,beam }}\left(\omega^{\prime}, \gamma^{\prime}\right)$ from Eq. (18) and replacing $\left(\omega^{\prime}, \gamma^{\prime}\right)$ by the appropriate parameters $(\omega, \gamma)$ and $(\Omega, \Gamma)$ the latter quantities are expressed as

$$
\begin{aligned}
f_{1}(\omega)= & \left(\left(d^{\text {iso }}(\omega)\right)^{2}-\left(d^{\text {ani }}(\omega)\right)^{2}\right) e^{-\frac{(t-\tau)^{2}}{\gamma^{2}}} e^{2 i \omega t} \\
& \times\left(\left(E_{0 x}^{\text {probe }}\right)^{2}+\left(E_{0 y}^{\text {probe }}\right)^{2}\right), \\
f_{2}(\Omega)= & \left(d^{\text {iso }}(\Omega) d^{\text {iso }}(-\Omega)-d^{\text {ani }}(\Omega) d^{\text {ani }}(-\Omega)\right) e^{-\frac{t^{2}}{\Gamma^{2}}} \\
& \times\left(\left(E_{0 x}^{\text {pump }}\right)^{2}+\left(E_{0 y}^{\text {pump }}\right)^{2}\right) .
\end{aligned}
$$

By plugging Eqs. (21) and (22) into Eq. (16), the third-order equation Eq. (13) is modified into

$$
\begin{aligned}
\ddot{\mathbf{r}}_{b}^{(3)}+\frac{\dot{\mathbf{r}}_{b}^{(3)}}{\tau_{b}}+\omega_{0}^{2} \mathbf{r}_{b}^{(3)}= & 3 b f_{1}(\omega) \mathbf{r}^{(1), \text { probe }}(-\omega) \\
& +6 b f_{2}(\Omega) \mathbf{r}^{(1), \text { probe }}(\omega) \\
& -\frac{e \mathbf{P}_{b}^{(3)}}{m \epsilon_{0}}-\frac{e}{m} \dot{\mathbf{r}}_{b}^{(3)} \wedge \mathbf{B}_{\text {mat }} .
\end{aligned}
$$

Then, by summing over all the $N_{b}$ electrons, by evaluating the macroscopic third-order polarization $\mathbf{P}_{b}^{(3)}=\frac{1}{V} \sum_{i}^{N_{b}}-e \mathbf{r}_{b_{i}}^{(3)}$, and by writing $\mathbf{r}^{(1) \text {,probe }}(-\omega)$ and $\mathbf{r}^{(1) \text {,probe }}(\omega)$ with the help of Eq. (18), Eq. (23) is transformed into 


$$
\begin{aligned}
\mathbf{P}_{b}^{(3)}= & \epsilon_{0} \chi_{b}^{(3)}(\omega, \gamma) g^{\text {probe }}(t, \tau)\left[\begin{array}{cc}
d^{\text {iso }}(-\omega) & -i d^{\text {ani }}(-\omega) \\
i d^{\text {ani }}(-\omega) & d^{\text {iso }}(-\omega)
\end{array}\right] \mathbf{E}^{\text {probe }} \\
& +\epsilon_{0} \chi_{b}^{(3), \text { pump-probe }}(\omega, \gamma, \Omega, \Gamma) g^{\text {pump }}(t)\left[\begin{array}{cc}
d^{\text {iso }}(\omega) & -i d^{\text {ani }}(\omega) \\
i d^{\text {ani }}(\omega) & d^{\text {iso }}(\omega)
\end{array}\right] \mathbf{E}^{\text {probe }}-i \xi_{b} \mathbf{P}_{b}^{(3)} \wedge \mathbf{e}_{z}
\end{aligned}
$$

where $g^{\text {probe }}(t, \tau)=e^{-\frac{(t-\tau)^{2}}{\gamma^{2}}}\left(\left(E_{0 x}^{\text {probe }}\right)^{2}+\left(E_{0 y}^{\text {probe }}\right)^{2}\right), g^{\text {pump }}(t)=e^{-\frac{t^{2}}{\Gamma^{2}}}\left(\left(E_{0 x}^{\text {pump }}\right)^{2}+\left(E_{0 y}^{\text {pump }}\right)^{2}\right) \quad$ and $\quad \chi_{b}^{(3)}(\omega, \gamma), \chi_{b}^{(3), \text { pump-probe }}$ $(\omega, \gamma, \Omega, \Gamma)$ are given by

$$
\begin{aligned}
\chi_{b}^{(3)}(\omega, \gamma) & =\frac{-3 e b \mathfrak{r}_{b}}{D_{b}(\omega, \gamma) \epsilon_{0}}\left(\left(d^{\text {iso }}(\omega)\right)^{2}-\left(d^{\text {ani }}(\omega)\right)^{2}\right) \\
\chi_{b}^{(3), \text { pump-probe }}(\omega, \gamma, \Omega, \Gamma) & =\frac{-6 e b \mathfrak{r}_{b}}{D_{b}(\omega, \gamma) \epsilon_{0}}\left(d^{\text {iso }}(\Omega) d^{\text {iso }}(-\Omega)-d^{\text {ani }}(\Omega) d^{\text {ani }}(-\Omega)\right) .
\end{aligned}
$$

It is now easy to express the third-order polarization in a tensorial form. Defining $\alpha_{b}^{(3)}=\frac{\chi_{b}^{(3)}(\omega, \gamma)}{1-\xi_{b}^{2}}$ and $\alpha_{b}^{(3) \text {,pump-probe }}(\omega, \gamma, \Omega, \Gamma)$ $=\frac{\chi_{b}^{(3) \text { pump probe }}(\omega, \gamma, \Omega, \Gamma)}{1-\xi_{b}^{2}(\omega)}$, Eq. (24) may be expressed as

$$
\begin{aligned}
\mathbf{P}_{b}^{(3)}= & \epsilon_{0} \alpha_{b}^{(3)} g^{\text {probe }}(t, \tau)\left[\begin{array}{lll}
\left(d^{\text {iso }}(-\omega)+\xi_{b} d^{\text {ani }}(-\omega)\right) & -i\left(d^{\text {ani }}(-\omega)+\xi_{b} d^{\text {iso }}(-\omega)\right) & 0 \\
i\left(d^{\text {ani }}(-\omega)+\xi_{b} d^{\text {iso }}(-\omega)\right) & \left(d^{\text {iso }}(-\omega)+\xi_{b} d^{\text {ani }}(-\omega)\right) & 0 \\
0 & 0 & X
\end{array}\right] \mathbf{E}^{\text {probe }} \\
& +\epsilon_{0} \alpha_{b}^{(3), \text { pump-probe }}(\omega, \gamma, \Omega, \Gamma) g^{\text {pump }}(t)\left[\begin{array}{lll}
\left(d^{\text {iso }}+\xi_{b} d^{\text {ani }}\right) & -i\left(d^{\text {ani }}+\xi_{b} d^{\text {iso }}\right) & 0 \\
i\left(d^{\text {ani }}+\xi_{b} d^{\text {iso }}\right) & \left(d^{\text {iso }}+\xi_{b} d^{\text {ani }}\right) & 0 \\
0 & 0 & X
\end{array}\right] \mathbf{E}^{\text {probe }} .
\end{aligned}
$$

In the above equation, we have mentioned when the functions depend on $-\omega$ or $\Omega$. If the dependence is not mentioned, it implicitly means that the parameter is the pulsation $\omega$.

\section{Metallic part-free electrons}

The polarization due to the free charge carriers is much easier to determine. By writing $\mathbf{r}_{f}(t)=\mathbf{r}_{f}^{(1)}(t)+\mathbf{r}_{f}^{(3)}(t)$, Eq. (10) leads to the following equations:

$$
\begin{aligned}
& \ddot{\mathbf{r}}_{f}^{(1)}+\frac{1}{\tau_{f}} \dot{\mathbf{r}}_{f}^{(1)}=-\frac{e \mathbf{E}^{\text {probe }}}{m_{f}}-\frac{e \mathbf{P}_{f}^{(1)}}{m_{f} \epsilon_{0}}-\frac{e \mathbf{P}_{b}^{(1)}}{m_{f} \epsilon_{0}}-\frac{e \dot{\mathbf{r}}_{f}^{(1)}}{m_{f}} \wedge \mathbf{B}_{\text {mat }} \\
& \ddot{\mathbf{r}}_{f}^{(3)}+\frac{1}{\tau_{f}} \dot{\mathbf{r}}_{f}^{(3)}=-\frac{e \mathbf{P}_{b}^{(3)}}{m_{f} \epsilon_{0}}-\frac{e \mathbf{P}_{f}^{(3)}}{m_{f} \epsilon_{0}}-\frac{e \dot{\mathbf{r}}_{f}^{(3)}}{m_{f}} \wedge \mathbf{B}_{\mathrm{mat}} .
\end{aligned}
$$

As before one sums over all the $N_{f}$ free electrons in the Fourier space with $\mathbf{P}_{f}^{(1)}=\frac{1}{V} \sum_{i}^{N_{f}}-e \mathbf{r}_{f_{i}}^{(1)}$ and $\mathbf{P}_{f}^{(3)}=\frac{1}{V} \sum_{i}^{N_{f}}-e \mathbf{r}_{f_{i}}^{(3)}$. This leads to

$$
\mathbf{P}_{f}^{(1)}=\epsilon_{0} \chi_{f}^{(1)} \mathbf{E}^{\text {probe }}-i \xi_{f} \mathbf{P}_{f}^{(1)} \wedge \mathbf{e}_{z}+\chi_{f}^{(1)} \mathbf{P}_{b}^{(1)}
$$

and

$$
\mathbf{P}_{f}^{(3)}=\chi_{f}^{(1)} \mathbf{P}_{b}^{(3)}-i \xi_{f} \mathbf{P}_{f}^{(3)} \wedge \mathbf{e}_{z},
$$

where $\chi_{f}^{(1)}=\chi_{f}^{(1)}(\omega, \gamma)=\frac{n_{i} e^{2}}{m_{f} D_{f}(\omega, \gamma) \epsilon_{0}}, \xi_{f}(\omega, \gamma)=\frac{e B_{\operatorname{mat}}\left(\omega+\frac{i t}{\gamma^{2}}\right)}{m_{f} D_{f}(\omega, \gamma)}$ with $D_{f} \equiv D_{f}(\omega, \gamma)=\left[\left(\frac{t^{2}}{\gamma^{4}}-\frac{1}{\gamma^{2}}-\frac{t}{\tau_{f} \gamma^{2}}-\frac{n_{i} e^{2}}{m_{f} \epsilon_{0}}-\omega^{2}\right)+i\left(\frac{\omega}{\tau_{f}}-\frac{2 \omega t}{\gamma^{2}}\right)\right]$. Then, using the expressions of $\mathbf{P}_{b}^{(1)}$ and $\mathbf{P}_{b}^{(3)}$ given by Eqs. (15) and (26) and defining $\alpha_{f} \equiv \alpha_{f}^{(1)}(\omega, \gamma)=\frac{\chi_{f}^{(1)}(\omega, \gamma)}{1-\xi_{f}^{2}(\omega, \gamma)}$, one obtains

$$
\mathbf{P}_{f}^{(1)}=\epsilon_{0} \alpha_{f}\left[\begin{array}{ccc}
1 & -i \xi_{f} & 0 \\
i \xi_{f} & 1 & 0 \\
0 & 0 & X
\end{array}\right] \mathbf{E}^{\text {probe }}+\epsilon_{0} \alpha_{b} \alpha_{f}\left[\begin{array}{ccc}
\left(1+\xi_{b} \xi_{f}\right) & -i\left(\xi_{b}+\xi_{f}\right) & 0 \\
i\left(\xi_{b}+\xi_{f}\right) & \left(1+\xi_{b} \xi_{f}\right) & 0 \\
0 & 0 & X
\end{array}\right] \mathbf{E}^{\text {probe }}
$$




$$
\begin{aligned}
\mathbf{P}_{f}^{(3)}= & \epsilon_{0} \alpha_{f}^{(1)} \alpha_{b}^{(3)} g^{\text {probe }}(t, \tau) \\
& \times\left[\begin{array}{ccc}
\left(d^{\text {iso }}(-\omega)\left(1+\xi_{b} \xi_{f}\right)+d^{\text {ani }}(-\omega)\left(\xi_{b}+\xi_{f}\right)\right) & -i\left(d^{\text {ani }}(-\omega)\left(1+\xi_{b} \xi_{f}\right)+d^{\text {iso }}(-\omega)\left(\xi_{b}+\xi_{f}\right)\right) & 0 \\
i\left(d^{\text {ani }}(-\omega)\left(1+\xi_{b} \xi_{f}\right)+d^{\text {iso }}(-\omega)\left(\xi_{b}+\xi_{f}\right)\right) & \left(d^{\text {iso }}(-\omega)\left(1+\xi_{b} \xi_{f}\right)+d^{\text {ani }}(-\omega)\left(\xi_{b}+\xi_{f}\right)\right) & 0 \\
0 & 0 & X
\end{array}\right] \mathbf{E}^{\text {probe }} \\
& +\epsilon_{0} \alpha_{f}^{(1)} \alpha_{b}^{(3), \text { pump-probe }}(\omega, \gamma, \Omega, \Gamma) g^{\text {pump }}(t) \\
& \times\left[\begin{array}{ccc}
\left(d^{\text {iso }}\left(1+\xi_{b} \xi_{f}\right)+d^{\text {ani }}\left(\xi_{b}+\xi_{f}\right)\right) & -i\left(d^{\text {ani }}\left(1+\xi_{b} \xi_{f}\right)+d^{\text {iso }}\left(\xi_{b}+\xi_{f}\right)\right) & 0 \\
i\left(d^{\text {ani }}\left(1+\xi_{b} \xi_{f}\right)+d^{\text {iso }}\left(\xi_{b}+\xi_{f}\right)\right) & \left(d^{\text {iso }}\left(1+\xi_{b} \xi_{f}\right)+d^{\text {ani }}\left(\xi_{b}+\xi_{f}\right)\right) & 0 \\
0 & 0 & X
\end{array}\right] \mathbf{E}^{\text {probe }} .
\end{aligned}
$$

\section{Response functions}

With $\mathbf{D}=\epsilon_{0} \mathbf{E}+\mathbf{P}$ and Eqs. (15), (26), (30), and (31), one can now identify the tensors elements $\epsilon_{i j}, \epsilon_{i j}^{(3)}$ and $\epsilon_{\text {probe } i j}^{\text {pump }}$ of Eqs. (4) and (6). The linear tensors elements remain identical to the ones in our previous work

$$
\begin{gathered}
\epsilon_{x x}=1+\alpha_{b}+\alpha_{f}+\alpha_{f} \alpha_{b}\left(1+\xi_{b} \xi_{f}\right), \\
\epsilon_{x y}=-i\left[\xi_{b} \alpha_{b}+\xi_{f} \alpha_{f}+\alpha_{b} \alpha_{f}\left(\xi_{b}+\xi_{f}\right)\right] .
\end{gathered}
$$

The tensor elements responsible for the third-order response are given by the following expressions:

$$
\begin{aligned}
\epsilon_{x x}^{(3)}= & \alpha_{b}^{(3)}\left[\left(d^{\mathrm{iso}}(-\omega)+\xi_{b} d^{\mathrm{ani}}(-\omega)\right)\right. \\
& \left.+\alpha_{f}^{(1)}\left(d^{\mathrm{iso}}(-\omega)\left(1+\xi_{b} \xi_{f}\right)+d^{\mathrm{ani}}(-\omega)\left(\xi_{b}+\xi_{f}\right)\right)\right], \\
\epsilon_{x y}^{(3)}= & -i \alpha_{b}{ }^{(3)}\left[\left(d^{\mathrm{ani}}(-\omega)+\xi_{b} d^{\text {iso }}(-\omega)\right)\right. \\
& \left.+\alpha_{f}^{(1)}\left(d^{\mathrm{ani}}(-\omega)\left(1+\xi_{b} \xi_{f}\right)+d^{\mathrm{iso}}(-\omega)\left(\xi_{b}+\xi_{f}\right)\right)\right] .
\end{aligned}
$$

They are slightly different from the ones in Ref. 17 due to the presence of the term $d^{\text {ani }}$. In the present work, we have taken into account the anisotropic displacement in the calculation of the nonlinear term $b \mathbf{r}^{3}$.

Finally, the tensor elements related to the pump-probe interaction are

$$
\begin{aligned}
\epsilon_{\text {probe } x x}^{\text {pump }}= & \alpha_{b}^{(3), \text { pump-probe }}(\omega, \gamma, \Omega, \Gamma)\left[\left(d^{\text {iso }}+\xi_{b} d^{\text {ani }}\right)\right. \\
& \left.+\alpha_{f}^{(1)}\left(d^{\text {iso }}\left(1+\xi_{b} \xi_{f}\right)+d^{\text {ani }}\left(\xi_{b}+\xi_{f}\right)\right)\right], \\
\epsilon_{\text {probe } x y}^{\text {pump }}= & -i \alpha_{b}^{(3) \text { pump-probe }}(\omega, \gamma, \Omega, \Gamma)\left[\left(d^{\text {ani }}+\xi_{b} d^{\text {iso }}\right)\right. \\
& \left.+\alpha_{f}^{(1)}\left(d^{\text {ani }}\left(1+\xi_{b} \xi_{f}\right)+d^{\text {iso }}\left(\xi_{b}+\xi_{f}\right)\right)\right] .
\end{aligned}
$$

In what follows the parameters of the Drude-Voigt model will be taken as identical to the ones in Reference 17 . We remember that the $\omega_{0}$ pulsation is associated with the energy band gap between the electronic bands $3 d$ and $4 s$ of Ni with $\hbar \omega_{0}=4 \mathrm{eV} .^{21}$ It is assumed that $m_{f}=m_{b}=m_{e}$. In this case, one can write $x_{f} \omega_{p}^{2}=\frac{\mathfrak{n}_{f} e^{2}}{m_{f} \epsilon_{0}}, x_{b} \omega_{p}^{2}=\frac{\mathfrak{n}_{b} e^{2}}{m_{b} \epsilon_{0}}$ and the value of the plasma pulsation $\hbar \omega_{p}=4.87 \mathrm{eV}$ can be found in Ref. 40. We have $x_{b}=0.4$ and $x_{f}=0.6 .^{34,35,41}$ The damping factors $\tau_{b}$ and $\tau_{f}$ for bound and free charge carriers may be fixed at $10^{-15} \mathrm{~s}$ and $10^{-14} \mathrm{~s} .^{42,43}$ The nonlinear parameter $b$ is expressed as $b=\frac{\omega_{0}^{2}}{a^{2}},{ }^{39}$ where $a=2.48 \times 10^{-10}$, and $\mathrm{m}$ is the distance between two near neighbors in Ni lattice crystallizing in a fcc structure. The value of the effective magnetic field is $B_{\text {mat }}=510 \mathrm{~T}^{35}$

We propose to investigate the impact of the third-order terms $\epsilon^{(3)}$ and $\epsilon_{\text {probe }}^{\text {pump }}$ on the total nonlinear response functions $\epsilon^{\mathrm{nl}}$ and $\epsilon^{\mathrm{nlp}}$ regarding the amplitude of the external electric field. In the experiment presented in Fig. 1, the absorbed energy $\mathcal{E}_{a b s}$ was $0.4 \mathrm{~mJ} / \mathrm{cm}^{2}$ which corresponds to a pumpamplitude of $E=4 \times 10^{8} \mathrm{~V} / \mathrm{m}$ following the relation $\frac{\mathcal{E}_{a b s}}{\mathcal{A}}$ $=\frac{c \epsilon_{0} \Delta t E^{2}}{2}$, where $\Delta t$ is the pulse duration and $\mathcal{A}$ is the value of the absorption coefficient (see details in Ref. 17).

In Fig. 2, we present the real part of all the isotropic response functions $\operatorname{Re}\left(\epsilon_{x x}^{(k)}\right)$ as functions of the photon energy with a pump-probe time delay $\tau=0$ and the following electric field components $\mathbf{E}^{\text {probe }}=\left(E_{0 x}^{\text {probe }} \mathbf{e}_{x}, 0\right), \mathbf{E}^{\text {pump }}$ $=\left(E_{0 x}^{\text {pump }} \mathbf{e}_{x}, 0\right)$. We have worked with $\omega=\Omega$ and the parameters $\gamma$ and $\Gamma$ have been fixed at $25 \times 10^{-15} \mathrm{~s}$ in order to represent the 50-fs pulse durations. The black curve represents the linear term $\operatorname{Re}\left(\epsilon_{x x}\right)$, which must be considered as the reference. In the inset, one can see the third-order tensor element $\operatorname{Re}\left(\epsilon_{x x}^{(3)}\right)$ (blue line) and the pump-probe term $\operatorname{Re}\left(\epsilon_{\text {probe } x x}^{\text {pump }}\right)$ (red dashed line) whose amplitudes are very

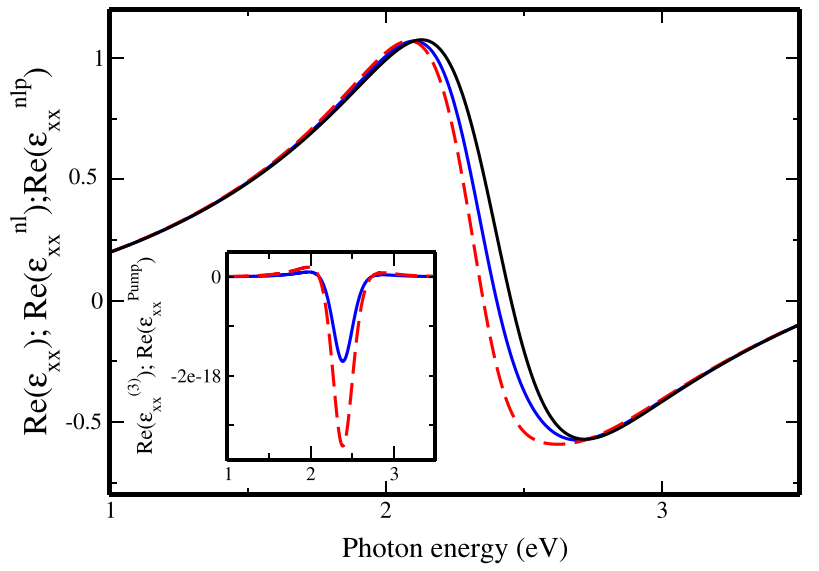

FIG. 2. $\operatorname{Re}\left(\epsilon_{x x}\right)$ (black line), $\operatorname{Re}\left(\epsilon_{x x}^{\mathrm{nl}}\right)$ with $\left\|\mathbf{E}^{\text {probe }}\right\|=4 \times 10^{8} \mathrm{~V} / \mathrm{m}$ (blue line) and $\operatorname{Re}\left(\epsilon_{x x}^{\text {nlp }}\right)$ with $\left\|\mathbf{E}^{\text {probe }}\right\| \approx 10^{7} \mathrm{~V} / \mathrm{m}$ and $\left\|\mathbf{E}^{\text {pump }}\right\|=4 \times 10^{8} \mathrm{~V} / \mathrm{m}$ (red-dashed line) as functions of the photon energy $(\mathrm{eV})$ with a time delay $\tau=0$ and $\omega=\Omega$. Inset: $\operatorname{Re}\left(\epsilon_{x x}^{(3)}\right)$ (blue line) and $\operatorname{Re}\left(\epsilon_{\text {probe }_{\text {prx }}}^{\text {pump }}\right)$ (red-dashed line) as functions of the photon energy $(\mathrm{eV})$. 
small $\left(\approx 10^{-18}\right)$ compared to $\operatorname{Re}\left(\epsilon_{x x}\right)$. They both contribute to the nonlinear responses with $\operatorname{Re}\left(\epsilon_{x x}^{\mathrm{nl}}\right)=\operatorname{Re}\left(\epsilon_{x x}\right)+\operatorname{Re}\left(\epsilon_{x x}^{(3)}\right)$ $\times\left(E_{0 x}^{\text {probe }}\right)^{2}$ and $\operatorname{Re}\left(\epsilon_{x x}^{\mathrm{nlp}}\right)=\operatorname{Re}\left(\epsilon_{x x}^{\mathrm{nl}}\right)+\operatorname{Re}\left(\epsilon_{\text {probe } x x}^{\text {pump }}\right) \times\left(E_{0 x}^{\text {pump }}\right)^{2}$. Hence, to reach the order of magnitude of the linear term $\operatorname{Re}\left(\epsilon_{x x}\right)$ (which is of the order of unity), the amplitude of the external electric fields must be on the order of $10^{9} \mathrm{~V} / \mathrm{m}$. As an illustration, the blue curve (in the main box of Fig. 2) represents $\operatorname{Re}\left(\epsilon_{x x}^{\mathrm{nl}}\right)$ with $\left\|\mathbf{E}^{\text {probe }}\right\|=4 \times 10^{8} \mathrm{~V} / \mathrm{m}$. Let us stress that almost no modifications are observed with $\left\|\mathbf{E}^{\text {probe }}\right\|$ $\approx 10^{7} \mathrm{~V} / \mathrm{m}$ (ratio $<10^{-4}$ ). This latest value can be taken as the value of the probe electric field since its contribution has to lie in the linear regime and its amplitude is generally a tenth of the pump one. Hence, the red-dashed curve (in the main box of Fig. 2) represents $\operatorname{Re}\left(\epsilon_{x x}^{\text {nlp }}\right)$ with $\left\|\mathbf{E}^{\text {probe }}\right\|$ $\approx 10^{7} \mathrm{~V} / \mathrm{m}$ and $\left\|\mathbf{E}^{\text {pump }}\right\|=4 \times 10^{8} \mathrm{~V} / \mathrm{m}$. Let us note that the time-dependent terms in the above functions have been neglected in order to calculate these static quantities.

This gives an idea of the nonlinear terms influence compared to the linear one. We mentioned that a similar behavior is observed with the imaginary part of the $\epsilon_{x x}^{(k)}$ functions and also with the real and imaginary parts of the anisotropic response functions $\epsilon_{x y}^{(k)}$ whose amplitudes were smaller $\left(\approx 10^{-1}\right)$. The apparition of the nonlinear regime $\left(E \approx 10^{8} \mathrm{~V} / \mathrm{m}\right)$ is coherent with the experimental results presented in Ref. 9 regarding the single-pulse-like Faraday experiment where the absorbed energy varied from $10^{-3} \mathrm{~mJ} / \mathrm{cm}^{2}$ to $1 \mathrm{~mJ} / \mathrm{cm}^{2}$ corresponding to an electric field amplitude between $10^{7} \mathrm{~V} / \mathrm{m}$ and $10^{9} \mathrm{~V} / \mathrm{m}$.

Let us return to the magneto-optical rotation calculation. It appears that the present model is unsuccessful to describe the results presented in Fig. 1. Indeed the influence of the pump electric field enters only through the term $g^{\text {pump }}(t)$ $=e^{-\frac{t^{2}}{\Gamma^{2}}}\left(\left(E_{0 x}^{\text {pump }}\right)^{2}+\left(E_{0 y}^{\text {pump }}\right)^{2}\right)$, which is quadratic and working in the configuration $\mathbf{E}_{0}^{\text {pump }}=\left(E_{0}^{\text {pump }} \mathbf{e}_{x}, 0\right)=$ or $\mathbf{E}_{0}^{\text {pump }}=\left(0, E_{0}^{\text {pump }} \mathbf{e}_{y}\right)$ will give the same contribution in the calculation of the rotation angle (see also Section IV).

The result of Bigot and coworkers suggests that the pump electric field creates an anisotropy which is different depending on the polarization directions. In the Drude-Voigt model, the anisotropic aspect is represented by the Lorentz magnetic force $-e \dot{\mathbf{r}} \wedge \mathbf{B}_{\text {mat }}$. We thus propose to incorporate this ingredient in order to build a light-induced Weiss molecular mean-field model.

\section{Light-induced coherent molecular mean-field}

Following the results of Fig. 1, it seems reasonable to suggest that an ultrafast laser pulse modifies the magnetic properties of a ferromagnetic sample and creates a coherent induced-magnetic field depending on its amplitude and its polarization. We propose to define this quantity $\mathbf{B}_{\text {mat }}(\mathbf{E})$ as

$$
\mathbf{B}_{\text {mat }}(\mathbf{E})=\mathbf{B}_{\text {mat }}+\lambda^{\text {Weiss }} f(\mathbf{E}),
$$

where $\lambda^{\text {Weiss }} f(\mathbf{E})$ is a light-induced molecular mean-field that adds to the static molecular mean-field. The parameter $\lambda^{\text {Weiss }}$ is a mean parameter, which could be calculated in different ways incorporating various mechanisms, and $f(\mathbf{E})$ is a vectorial function involving the external electric field. Nevertheless, the quantity $f(\mathbf{E})$ needs to obey some constraints. The components of the electric field belong to the plane defined by $\left(\mathbf{e}_{x}, \mathbf{e}_{y}\right)$ whereas the magnetic field remains in the $\mathbf{e}_{z}$ direction. The experimental results show that excitation in the $\mathbf{e}_{x}$ or $\mathbf{e}_{y}$ axis does not produce the same effect. This suggests that the function $f(\mathbf{E})$ could be represented by a vectorial product.

\section{Study of coherent relativistic spin-orbit terms}

To this end, the SOC of Eq. (1) presents interesting directions for further investigations. Let us write the electromagnetic field inside the material (mean field produced by charges and spins of the material) as $\left(\mathbf{E}_{\text {mat }}, \mathbf{A}_{\text {mat }}\right)$ while the ones carried out by the light are kept as $(\mathbf{E}, \mathbf{A})$. The term $\mathbf{E} \wedge(\mathbf{p}+e \mathbf{A})$ leads to the following possible interactions: $\mathbf{E} \wedge \mathbf{p}, \mathbf{E} \wedge\left(e \mathbf{A}_{\text {mat }}\right), \mathbf{E}_{\text {mat }} \wedge\left(e \mathbf{A}_{\text {mat }}\right), \mathbf{E}_{\text {mat }} \wedge \mathbf{p}$, and $\mathbf{E}_{\text {mat }} \wedge(e \mathbf{A})$. If one focuses only on the terms induced by the external laser field, one may write the SOC as

$$
-\frac{e \hbar}{4 m_{e} c^{2}} \boldsymbol{\sigma} \cdot\left(\mathbf{E} \wedge \mathbf{p}+\mathbf{E}_{\mathrm{mat}} \wedge e \mathbf{A}+\mathbf{E} \wedge e \mathbf{A}_{\mathrm{mat}}\right) .
$$

Let us emphasize that $\mathbf{E}_{\text {mat }} \wedge e \mathbf{A}_{\text {mat }}$ and $\mathbf{E}_{\text {mat }} \wedge \mathbf{p}$ are not considered here since they do not explicitly involve the laser field. Considering the previous interaction, one can write the Zeeman energy for any electron spin as

$$
\begin{aligned}
E_{Z}= & \frac{e \hbar}{2 m} \boldsymbol{\sigma} \cdot \mathbf{B}_{\text {mat }}(\mathbf{E}) \\
= & \frac{e \hbar}{2 m} \boldsymbol{\sigma} \cdot\left(\mathbf{B}_{\text {mat }}+\frac{1}{2 m c^{2}} \mathbf{E} \wedge\left(e \mathbf{A}_{\text {mat }}\right)\right. \\
& \left.+\frac{1}{2 m c^{2}} \mathbf{E}_{\text {mat }} \wedge(e \mathbf{A})+\frac{1}{2 m c^{2}} \mathbf{E} \wedge \mathbf{p}\right),
\end{aligned}
$$

where $\mathbf{B}_{\text {mat }}$ is the Weiss molecular mean-field and the second term in Eq. (40) is the effective field seen by an electron interacting via the SOC (we neglect the laser magnetic field here). The latter may reasonably play the role of $\lambda^{\text {Weiss }} f(\mathbf{E})$ and its components read

$$
\begin{aligned}
& \mathbf{B}_{\mathrm{mat}}(\mathbf{E})= \mathbf{B}_{\mathrm{mat}}+\frac{e}{2 m c^{2}} \mid \begin{array}{c}
E_{y} A_{\mathrm{mat}_{z}} \\
-E_{x} A_{\mathrm{mat}_{z}} \\
E_{x} A_{\mathrm{mat}_{y}}-E_{y} A_{\mathrm{mat}_{x}}
\end{array} \\
&+\frac{e}{2 m c^{2}} \mid \begin{array}{c}
-E_{\mathrm{mat}_{z}} A_{y} \\
E_{\mathrm{mat}_{z}} A_{x} \\
E_{x \mathrm{mat}} A_{y}-E_{y \mathrm{mat}} A_{x}
\end{array} \\
&+\frac{1}{2 m c^{2}} \mid \begin{array}{c}
0 \\
0 \\
E_{x} p_{y}-E_{y} p_{x} .
\end{array}
\end{aligned}
$$

In the presence of a static magnetic field, a dimensional analysis allows us to write the static vector potential as $\mathbf{A}_{\text {mat }}$ $=\left(a_{\mathrm{Ni}} B_{\mathrm{mat}} \mathbf{e}_{x}, a_{\mathrm{Ni}} B_{\text {mat }} \mathbf{e}_{y}, 0\right)$, where $a_{\mathrm{Ni}}$ denotes the mean atomic radius of a nickel valence electron. In the same way, we assume that the internal electric field is related to the internal magnetic field with the following dimensional analysis: $\mathbf{E}_{\text {mat }}=\left(\left\langle v_{0}\right\rangle B_{\text {mat }} \mathbf{e}_{x},\left\langle v_{0}\right\rangle B_{\text {mat }} \mathbf{e}_{y},\left\langle v_{0}\right\rangle B_{\text {mat }} \mathbf{e}_{z}\right)$, where 
$\left\langle v_{0}\right\rangle$ is a characteristic mean electron velocity. The electron momenta may be also written as $\left|p_{x}\right| \approx\left|p_{y}\right| \approx m_{e}\left\langle v_{0}\right\rangle$. The potential vector of the light is $\mathbf{A}=-\frac{1}{\omega}\left(E_{x} \mathbf{e}_{x}, E_{y} \mathbf{e}_{y}, 0\right)$ since $\mathbf{E}=-\frac{\partial \mathbf{A}}{\partial t}$. Following this assumption and focusing on the $z$ components, Eq. (41) is transformed into

$$
\begin{aligned}
& \mathbf{B}_{\text {mat }}(\mathbf{E}) \approx \mathbf{B}_{\text {mat }}-B_{\text {mat }} \times \underbrace{\frac{e a_{\mathrm{Ni}}}{2 m c^{2}}}_{\lambda_{1}}
\end{aligned}
$$

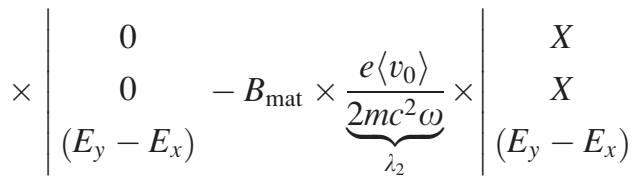

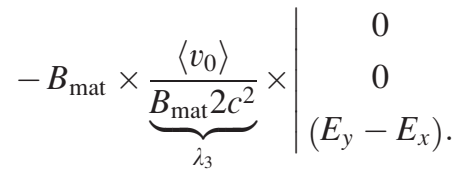

The above equation exhibits an interesting form since the internal magnetic field is enhanced or reduced depending on the polarization of the electric field. The light-modified internal magnetic field may also be expressed as

$$
\mathbf{B}_{\text {mat }}(\mathbf{E})=B_{\text {mat }}\left(1-\left(\lambda_{1}+\lambda_{2}+\lambda_{3}\right)\left(E_{y}-E_{x}\right)\right) \mathbf{e}_{z},
$$

where $\lambda_{1}=\frac{e a_{\mathrm{Ni}}}{2 m c^{2}}, \lambda_{2}=\frac{e\left\langle v_{0}\right\rangle}{2 m c^{2} \omega}$, and $\lambda_{3}=\frac{\left\langle v_{0}\right\rangle}{B_{\mathrm{mat}} 2 c^{2}}$. With $a_{\mathrm{Ni}}$ $\approx 10^{-10} \mathrm{~m}, \lambda_{1}=\frac{1.6 \times 10^{-19} \times 10^{-10}}{2 \times 10^{-30} \times\left(3 \times 10^{8}\right)^{2}} \approx 8.8 \times 10^{-17} \mathrm{~m} / \mathrm{V}$. In the framework of the Drude model, $\left\langle v_{0}\right\rangle$ is evaluated with $\left\langle v_{0}\right\rangle$ $=|\dot{\mathbf{r}}| \approx \frac{\omega e E}{m \omega_{0}^{2}} \approx 4097 \mathrm{~m} / \mathrm{s}$ by taking $\omega=2.36 \times 10^{15} \mathrm{rad} / \mathrm{s}, \omega_{0}$ $=6.4 \times 10^{15} \mathrm{rad} / \mathrm{s}, \mathbf{E}=4 \times 10^{8} \mathrm{~V} / \mathrm{m}$, leading to $\lambda_{2}=1.54$ $\times 10^{-18} \mathrm{~m} / \mathrm{V}$ and $\lambda_{3}=4.46 \times 10^{-17} \mathrm{~m} / \mathrm{V}$ with $B_{\text {mat }}=510 \mathrm{~T}$.

The importance of this phenomenon thus depends only on the amplitude of the electric field. As an example for the first term (which is the largest), one has to compare $\zeta=\lambda_{1} E_{y}=\frac{e a_{\mathrm{Ni}} E_{y}}{2 m c^{2}}$ (or $E_{x}$ ) to unity. Unfortunately, the latter quantity appears to be very small. Indeed, with $E_{x} \approx E_{y}$ $\approx 10^{8} \mathrm{~V} / \mathrm{m}$, one obtains $\zeta=8.8 \times 10^{-17} \times 10^{8}=8.8$ $\times 10^{-9}$. However, this value is based on a one-electron interaction while one should probably consider the effect of all the electrons within a mean-field description.

Let us perform a quick estimate by considering roughly the number of electrons irradiated by the laser pulse $N_{\text {ir }}$. The latter can be defined as $N_{\mathrm{ir}}=n_{\mathrm{Ni}} \times d^{2} \times L \times n_{\nu}$, where $n_{\mathrm{Ni}} \approx 10^{28}$ atoms/ $\mathrm{m}^{3}$ is the nickel density, $L \approx 10^{-9} \mathrm{~m}$ is the thickness of the film, $d \approx 10^{-6} \mathrm{~m}$ is the beam spot diameter of the laser pulse, and $n_{\nu}$ is the number of photons absorbed by a nickel atom, which can be taken as $n_{\nu}=0.01$ according to Reference 44 . In that case, one gets $N_{\mathrm{ir}} \approx 10^{7}$ and $\lambda_{1}$ (resp. $\zeta$ ) becomes $\lambda_{1}^{\prime}=N_{\mathrm{ir}} \lambda_{1}=8.8 \times 10^{-10} \mathrm{~m} / \mathrm{V}$ (resp. $\zeta^{\prime}=N_{\mathrm{ir}} \zeta \approx 8.8 \times 10^{-2}$ ), suggesting that the effect could be much bigger.

Let us stress that this estimate needs to be performed in a more rigorous and sophisticated manner. For instance, the values of $d$ and $L$ are larger in Ref. 9 and lead to a value of $\zeta^{\prime}$ $10^{3}$ larger. Moreover, the estimate of $n_{\nu}$ may also change depending on the sample or on the irradiation conditions. Also, summing over all electrons may lead to an estimate that is too large in comparison to the one obtained using a proper mean-field description. This work may be achieved with the help of a self-consistent mean-field model, including all the light-matter interaction operators up to the secondorder in $1 / m_{e}$. One part of that work was already achieved in Refs. 26 and 27 by considering the relativistic interactions (up to the second-order in $1 / m_{e}$ ) between $N$ interacting electrons. The addition of an external time-dependent electromagnetic field in the above mentioned approach is currently under development and will enable us to tackle this issue in the near future.

\section{Phenomenological interaction}

However, the important aspect on which we propose to focus is the vectorial form obtained in Eq. (43), which involves the quantity $B_{\text {mat }}\left(E_{y}-E_{x}\right)$ in the $z$ direction. Hence, in order to circumvent the problem of the mechanism, we propose to keep only the physical ingredients of the previous discussion. We suggest working with the quantity $\lambda^{\text {Weiss }} f(\mathbf{E})$ postulated as

$$
\lambda^{\text {Weiss }} f(\mathbf{E}) \equiv B_{\text {mat }} \times \lambda \times\left(E_{y}-E_{x}\right) \mathbf{e}_{z},
$$

where $\lambda([\lambda]=\mathrm{m} / \mathrm{V})$ is a parameter underlying the meanperturbation induced by the external electric field. Let us note that the modification of the internal magnetic field by the external electric field is treated as a mean value and is not included within the classical theory of perturbation used to solve the Drude-Voigt equations. For a mechanism based on the previous SOC, one has $\lambda=\frac{e n_{i r} a_{\mathrm{Ni}}}{2 m c^{2}}=8.8 \times 10^{-10} \approx 10^{-9} \mathrm{~m} / \mathrm{V}$.

Consequently, according to Eq. (44), the magnetic field inside the material $\mathbf{B}_{\text {mat }}$ becomes $\mathbf{B}_{\text {mat }}(\mathbf{E})=\mathbf{B}_{\text {mat }}\left(1+\lambda E_{x}\right)$ with an electric field vibrating along the $\mathbf{e}_{x}$ axis and $\mathbf{B}_{\text {mat }}(\mathbf{E})=\mathbf{B}_{\text {mat }}\left(1-\lambda E_{y}\right)$ when the electric field vibrates along the $\mathbf{e}_{y}$ axis. If $E_{x}=E_{y}=E$, the difference between the two magnetic states will be $2 B_{\text {mat }} \lambda E \mathrm{~T}$. This shift increases with the amplitude of the external field.

In order to incorporate this modification in our model, one has to substitute $\mathbf{B}_{\text {mat }}$ by $\mathbf{B}_{\text {mat }}(\mathbf{E})$ in all the previous dielectric response functions. More precisely, the modification must be done in the functions $\xi_{b}$ and $\xi_{f}$ that are the only terms depending on $\mathbf{B}_{\text {mat }}$. When the latter depend on the probe electric field parameter $(\omega, \gamma) \mathbf{B}_{\text {mat }}$ is replaced by $\mathbf{B}_{\text {mat }}\left(\mathbf{E}^{\text {probe }}\right)$ whereas it is replaced by $\mathbf{B}_{\text {mat }}\left(\mathbf{E}^{\text {pump }}\right)$ in the functions depending on the pump parameters $(\Omega, \Gamma)$ (that is, only the case for functions described by Eqs. (36) and (37)).

In what follows, the value of the parameter $\lambda$ will be fixed at $\lambda=10^{-9} \mathrm{~m} / \mathrm{V}$. This choice is pertinent for the following reasons. The amplitude of the probe electric field is taken as $\left\|\mathbf{E}^{\text {probe }}\right\| \approx 10^{7} \mathrm{~V} / \mathrm{m}$, which leads to $\lambda E^{\text {probe }}=10^{-2}$. In the presence of the probe electric field, the magnetic field inside the material is thus modified less than one percent. Otherwise, with the value $\left\|\mathbf{E}^{\text {pump }}\right\|=4 \times 10^{8} \mathrm{~V} / \mathrm{m}$, as in the experiment of Ref. 9 , one gets $\lambda E^{\text {probe }}=0.4$ and the change of the magnetic state becomes more significant. Using the previous values, the theoretical calculations are presented in Section IV where the influence of the $\lambda$ parameter is also discussed. 


\section{RESULTS}

Our results are presented into two parts. In the next paragraph, we compare our theoretical predictions for the magneto-optical rotation angle with the experimental measurements presented in Section II. In the second part, we show, on the basis of our model, how the experimental results could be modified by changing the photon energy of the probe pulse.

\section{A. Comparison with experiment}

With the help of the tools developed in Section III, we can calculate the normalized rotation angle as $\frac{\Delta \theta(\tau)}{\theta}$ $=\frac{\theta_{\text {probe }}^{\text {pump }}(\tau)-\theta_{\text {alone }}^{\text {probe }}}{\theta_{\text {alone }}^{\text {probe }}}$ for the two configurations: $\theta_{\mathrm{PP}=0^{\circ}}$ where the electric field of the probe is parallel to the one of the pump and $\theta_{\mathrm{PP}=90^{\circ}}$ when they are perpendicular. The quantities $\theta_{\text {alone }}^{\text {probe }}$ and $\theta_{\text {probe }}^{\text {pump }}(\tau)$ are calculated as follows. The electric field of the probe is oriented along the $\mathbf{e}_{x}$ axis $\mathbf{E}^{\text {probe }}$ $=\left(E_{0 x}^{\text {probe }} \mathbf{e}_{x}, 0\right)$ and the components of the outgoing electric field given by Eq. (7) read $\mathbf{E}^{\text {out }}=\left(\epsilon_{x x}^{\mathrm{nl}} E_{0 x}^{\mathrm{probe}} \mathbf{e}_{x}, \epsilon_{x y}^{\mathrm{nl}} E_{0 x}^{\mathrm{probe}} \mathbf{e}_{y}\right)$. The latter is plugged into Eq. (2) and the associated static magneto-optical rotation $\theta^{\text {nl }}$ is calculated by using Eq. (3). Thus, one obtains $\theta_{\text {alone }}^{\text {probe }} \equiv \theta^{\text {nl }}$.

The same procedure is used to calculate the magnetooptical rotation angle in a pump-probe configuration. The dielectric tensor elements $\epsilon_{i j}^{\mathrm{nl}}$ are replaced by $\epsilon_{i j}^{\mathrm{nlp}}$, which modifies the magneto-optical rotation angle and gives $\theta_{\text {probe }}^{\text {pump }}(\tau)$ $\equiv \theta^{\text {nlp }}$. In the parallel configuration, one has $\mathbf{E}^{\text {pump }}$ $=\left(E_{0 x}^{\text {pump }} \mathbf{e}_{x}, 0\right)$ and the internal magnetic field is written as $\mathbf{B}_{\text {mat }}\left(\mathbf{E}_{0 x}^{\text {pump }}\right)=\mathbf{B}_{\text {mat }}\left(1+\lambda E_{0 x}^{\text {pump }}\right)$, whereas in the perpendicular configuration, one has $\mathbf{E}^{\text {pump }}=\left(0, E_{0 y}^{\text {pump }} \mathbf{e}_{y}\right)$ and $\mathbf{B}_{\text {mat }}\left(\mathbf{E}_{0 y}^{\text {pump }}\right)=\mathbf{B}_{\text {mat }}\left(1-\lambda E_{0 y}^{\text {pump }}\right)$. Therefore, one uses $\theta_{\|}^{\text {nlp }}$ and $\theta_{\perp}^{\text {nlp }}$ to denote the two configurations and one also defines the theoretical rotation angles $\theta_{\mathrm{th}}\left(\mathrm{PP}=0^{\circ}\right)$ and $\theta_{\text {th }}\left(\mathrm{PP}=90^{\circ}\right)$ as

$$
\begin{aligned}
& \theta_{\mathrm{th}}\left(\mathrm{PP}=0^{\circ}\right)=\frac{\Delta \theta(\tau)}{\theta_{\|}}=\frac{\theta_{\|}^{\mathrm{nlp}}-\theta^{(\mathrm{nl})}}{\theta^{\mathrm{nl}}}, \\
& \theta_{\mathrm{th}}\left(\mathrm{PP}=90^{\circ}\right)=\frac{\Delta \theta(\tau)}{\theta_{\perp}}=\frac{\theta_{\perp}^{\mathrm{nlp}}-\theta^{\mathrm{nl}}}{\theta^{\mathrm{nl}}} .
\end{aligned}
$$

The above theoretical quantities are compared with the experimental ones $\theta_{\exp }\left(\mathrm{PP}=0^{\circ}\right)$ and $\theta_{\exp }\left(\mathrm{PP}=90^{\circ}\right)$ already depicted in Fig. 1. Let us remember that the latter includes thermal effects which have been ignored in the present model. In agreement with the experiment in Ref. 9, the pulse parameters have been chosen as $\left\|\mathbf{E}^{\text {probe }}\right\|=10^{7} \mathrm{~V} / \mathrm{m}$, $\left\|\mathbf{E}^{\text {pump }}\right\|=4 \times 10^{8} \mathrm{~V} / \mathrm{m}, \quad \gamma=\Gamma=25 \times 10^{-15} \mathrm{~s}$, and $\hbar \omega$ $=\hbar \Omega=1.475 \mathrm{eV}$ corresponding to $50 \mathrm{fs}$ laser pulses centered at $\lambda_{l}=799 \mathrm{~nm}\left(\omega=2.36 \times 10^{15} \mathrm{rad} / \mathrm{s}\right)$. In order to investigate the dependence of the results with the lightinduced mean-field of Eq. (44), we also define the following rotation angle: $\theta_{\text {th }}(\operatorname{PP}(\lambda=0))$, where $\lambda=0$. The results are depicted in Fig. 3.

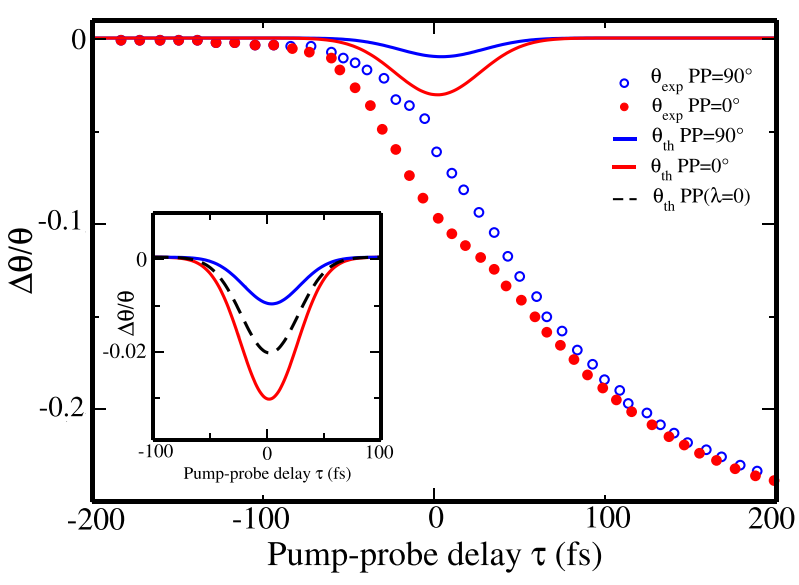

FIG. 3. Theoretical normalized coherent rotation angle $\theta_{\mathrm{th}}\left(\mathrm{PP}=0^{\circ}\right)$ and $\theta_{\text {th }}\left(\mathrm{PP}=90^{\circ}\right)$ for nickel as functions of the pump-probe delay $\tau(\hbar \omega=\hbar \Omega$ $=1.475 \mathrm{eV}$ ). Open and full circles represent the experimental normalized rotation angle (including thermal effects) taken from Ref. 9. Inset: $\theta_{\text {th }}\left(\mathrm{PP}=0^{\circ}\right), \theta_{\text {th }}\left(\mathrm{PP}=90^{\circ}\right)$, and $\theta_{\text {th }}(\operatorname{PP}(\lambda=0))$ as functions of the pumpprobe delay.

First of all, for time delays $\tau<0$, the theoretical coherent magneto-optical signals seem to follow the experimental trend until reaching a minimum at $\tau=0$. For positive delays $(\tau>0)$, contrary to what is experimentally observed, the theoretical results increase and return to zero. This finding is logical since we do not incorporate thermal effects and their associated relaxation phenomena which occur at longer timescales. Therefore, the present predictions could, in principle, reasonably represent the coherent rotation created by the laser pulses.

A noticeable difference is observed between $\theta_{\text {th }}\left(\mathrm{PP}=0^{\circ}\right)$ and $\theta_{\text {th }}\left(\mathrm{PP}=90^{\circ}\right)$ in the range $\tau \in[-100 \mathrm{fs}, 100 \mathrm{fs}]$, which may also be seen in the experimental signals. Although the amplitudes of the theoretical predictions are smaller than the experimental ones (mainly due to all the approximations used in the construction of the model), the calculated quantities are of the same order of magnitude and also have the same shapes. The difference has its origin in the differentiated action produced by the coherent molecular mean-field that we have introduced.

In the inset of Fig. 3, the previous coherent rotation angles $\theta_{\text {th }}\left(\mathrm{PP}=0^{\circ}\right)$ and $\theta_{\text {th }}\left(\mathrm{PP}=90^{\circ}\right)$ are compared to the one calculated with $\lambda=0: \theta_{\mathrm{th}}(\operatorname{PP}(\lambda=0))$. The latter is exactly the same in the parallel or perpendicular configurations and corresponds to the coherent magneto-optical rotation when the internal magnetic field $\mathbf{B}_{\text {mat }}$ remains constant.

In other words, $\theta_{\mathrm{th}}(\operatorname{PP}(\lambda=0))$ represents the nonlinear anisotropy created only by the electron charges, whereas $\theta_{\text {th }}\left(\mathrm{PP}=0^{\circ}\right)$ and $\theta_{\text {th }}\left(\mathrm{PP}=90^{\circ}\right)$ include also the modification of the internal magnetic field due to the interaction of the external electric fields with the electron spin degrees of freedom.

Let us now focus on the experimental results of Fig. 1(c) where the thermal effects are eliminated.

Since the difference between $\theta_{\text {th }}\left(\mathrm{PP}=0^{\circ}\right)$ and $\theta_{\text {th }}(\mathrm{PP}$ $\left.=90^{\circ}\right)$ is performed in the pump-probe configuration $\left(\theta_{\text {probe }}^{\text {pump }}(\tau)\right)$ and not with the normalized rotation $\Delta \theta / \theta$, one must calculate the coherent magnetic signal using 
$\theta_{\|}^{(\mathrm{nlp})}-\theta_{\perp}^{(\mathrm{nlp})}$. The results are depicted in Fig. 4 where the influence of $\lambda$ is shown.

It can be clearly seen on the figure that our theoretical predictions exhibit the appropriate order of magnitude as compared to the experimental results. Based on the assumptions of our model, it appears that the results are very sensitive to the value of $\lambda$. The results presented in Fig. 4 are obtained using $\lambda=10^{-9} \mathrm{~m} / \mathrm{V}$, and the value of the Kerr rotation angle at $\tau=0$ is around $-0.5 \mathrm{mrad}$. Let us stress that working with $\lambda=5 \times 10^{-10} \mathrm{~m} / \mathrm{V}$ and $\lambda=1.5 \times 10^{-9} \mathrm{~m} / \mathrm{V}$ leads, respectively, to a value of the Kerr rotation angle of $-0.25 \mathrm{mrad}$ and $-0.75 \mathrm{mrad}$. In that case, the theoretical predictions are too far from the experimental data and therefore not relevant. Due to the approximations inherent in the model, it would have been meaningless to extrapolate an accurate value of $\lambda$ related to the experimental signal. However, one may keep in mind the order of magnitude of $\lambda=10^{-9} \mathrm{~m} / \mathrm{V}$. Let us emphasize that the mechanism based on the SOC and the rough estimation of $n_{i r}$ calculated in Section III C lead to $\lambda=\frac{e a_{\mathrm{N} i} n_{i r}}{2 m c^{2}}=8.8 \times 10^{-10} \mathrm{~m} / \mathrm{V} \approx 9$ $\times 10^{-10} \mathrm{~V} / \mathrm{m}$. Due to all the approximations discussed in Section III C, an accurate analysis is absolutely necessary when considering the role and the importance of the SOC in coherent magneto-optical experiments. However, it has to be noted that, within the present semi-classical framework, the experimental trends are not reproduced when the parameter $\lambda$ is equal to zero. Before making some others interesting comments in Section $\mathrm{V}$, we propose to investigate how the latter results might be modified by changing the experimental parameters.

\section{B. Theoretical predictions on further experiments}

The possibility of performing similar experiments in a two-color scheme ${ }^{30}$ strongly encourage us to investigate the influence of the photon energy (or the frequency) of the laser pulses on the predictions of the model. A detailed study of the ultrafast coherent magneto-optical rotation in a two-color configuration is currently under progress and will soon be proposed for publication. In the following, we present some

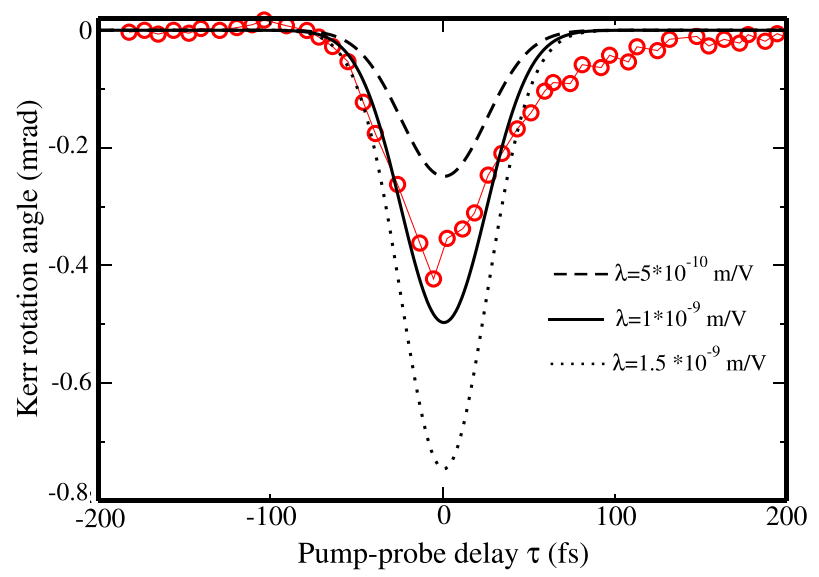

FIG. 4. Coherent magnetic signal for nickel as a function of the pump-probe delay $\tau(\hbar \omega=\hbar \Omega=1.475 \mathrm{eV})$. Open circles represent the experimental data taken from Ref. 9. interesting predictions concerning the experimental results quoted in Reference 9.

In Fig. 5, the value of the magneto-optical rotation angle at $\tau=0$ is depicted as a function of the probe photon energy $\hbar \omega$ for the static probe $\theta^{\text {probe }} \equiv \theta^{\text {nl }}$ and for the two pumpprobe configurations $\theta_{\|}^{(\mathrm{nlp})}$ and $\theta_{\perp}^{(\mathrm{nlp})}$.

One may distinguish four different areas labeled from I to IV where the relative positions between $\theta^{\text {probe }}, \theta_{\|}^{(\mathrm{nlp})}$, and $\theta_{\perp}^{(\mathrm{nlp})}$ are depicted.

They all follow the variation of the dielectric response functions. In area I, both pump-probe rotations are smaller than the static one and the perpendicular configuration has a higher value compared to the parallel configuration: $\theta_{\|}^{(\mathrm{nlp})}<\theta_{\perp}^{(\mathrm{nlp})}<\theta^{\text {probe }}$. Then, for higher photon energies, the parallel pump-probe rotation is smaller than the perpendicular pump-probe rotation and is observed in area II with $\theta_{\perp}^{(\mathrm{nlp})}<\theta_{\|}^{(\mathrm{nlp})}<\theta^{\text {probe }}$. A similar behavior is observed in regions III and IV where the pump-probe rotations are bigger than the static rotation corresponding, respectively, to the configurations $\theta_{\perp}^{(\mathrm{nlp})}<\theta^{\text {probe }}<\theta_{\|}^{(\mathrm{nlp})}$ and $\theta^{\text {probe }}<\theta_{\perp}^{(\mathrm{nlp})}$ $<\theta_{\|}^{(\mathrm{nlp})}$. It is worth mentioning that we have worked here with another pump photon energy $\hbar \Omega=2.4 \mathrm{eV}$ as compared to $\hbar \Omega=1.475 \mathrm{eV}$ used in Section III. This choice was done in order to obtain a better visibility of the presented results since the difference between the curves of Fig. 5 obtained with $\hbar \Omega=1.475 \mathrm{eV}$ are smaller.

As a consequence, their associated magneto-optical signals as functions of the time delay $\tau$ will exhibit clear differences. In Fig. 6, we present the normalized rotation angles as functions of the pump-probe time delay corresponding to the four previous configurations. The photon energies $\hbar \omega_{\mathrm{I}}=1.87 \mathrm{eV}, \hbar \omega_{\mathrm{II}}=2.1 \mathrm{eV}, \hbar \omega_{\mathrm{III}}=2.25 \mathrm{eV}$, and $\hbar \omega_{\mathrm{IV}}=2.35 \mathrm{eV}$ have been chosen for the sake of representational simplicity only.

The case I corresponds to the situation encountered in Reference 9 with $\theta_{\mathrm{PP}=0^{\circ}}<\theta_{\mathrm{PP}=90^{\circ}}$. Nevertheless, it would be possible to observe the opposite situation where $\theta_{\mathrm{PP}=0^{\circ}}$ $>\theta_{\mathrm{PP}=90^{\circ}}$ in areas II, III, and IV. Consequently, their associated coherent magnetic signals will exhibit positive values

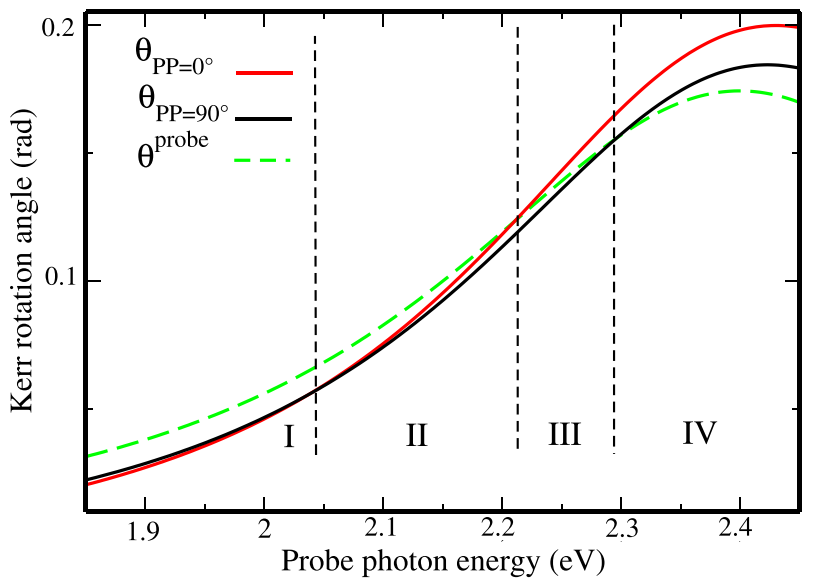

FIG. 5. Kerr rotation angles $\theta^{\text {probe }}, \theta_{\|}^{(\mathrm{nlp})}$, and $\theta_{\perp}^{(\mathrm{nlp})}$ at $\tau=0$ as functions of the probe photon energy. 


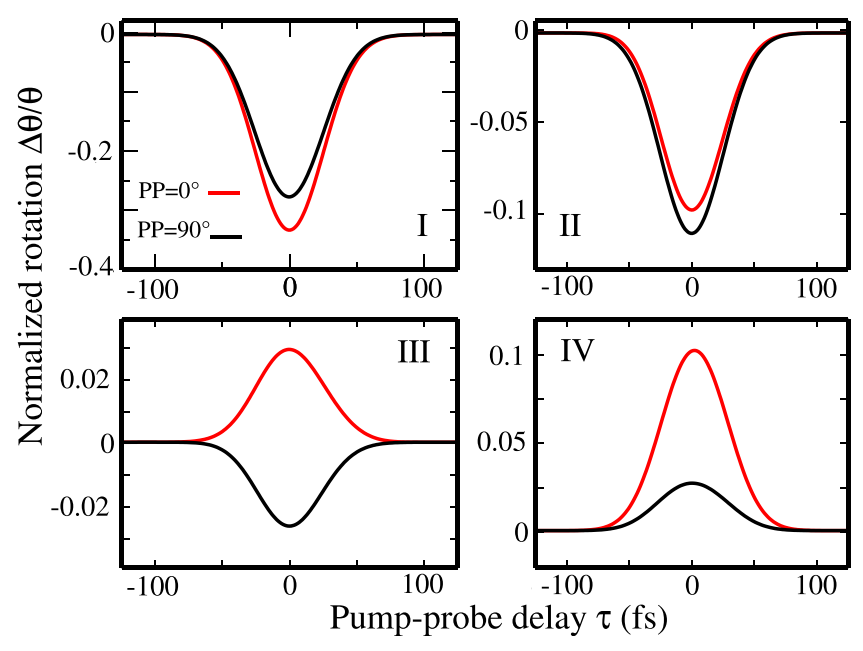

FIG. 6. Normalized rotation angles for nickel as functions of the pumpprobe delay $\tau$ for the probe photon energies $\hbar \omega_{\mathrm{I}}=1.87 \mathrm{eV}, \hbar \omega_{\mathrm{II}}=2.1 \mathrm{eV}$, $\hbar \omega_{\text {III }}=2.25 \mathrm{eV}$, and $\hbar \omega_{\mathrm{IV}}=2.35 \mathrm{eV}$. The pump photon energy is fixed at $\hbar \Omega=2.4 \mathrm{eV}$.

contrary to those presented in Fig. 4. Let us mention that the normalized rotation angles could also be positive. The most interesting case is probably the one of area III, where $\theta_{\mathrm{PP}=0^{\circ}}$ is positive whereas $\theta_{\mathrm{PP}=90^{\circ}}$ remains negative. In that case, by working with the appropriate experimental parameters, one may imagine that the total experimental magneto-optical signals (incorporating thermal effects) could exhibit different shapes compared to the ones depicted in Fig. 1(b), especially around $\tau=0$. This prediction may be useful for experimentalists.

\section{DISCUSSION AND CONCLUSIONS}

Here, we discuss the advantages and the limitations of the model. Some additional comments are also made.

First of all, the present model successfully reproduces the general trends of the coherent magneto-optical experiment performed by Bigot et al. ${ }^{9}$ By working with tabulated values of nickel physical properties, the theoretical predictions are of the same order of magnitude as available experimental data. The introduction of a phenomenological light-induced molecular mean-field, which depends on the electric fields polarizations, has enabled us to reproduce the difference between the parallel and the perpendicular configurations observed experimentally.

Moreover, it is worth mentioning that the comparison between our calculations and the experimental data is not rigorously perfect and some differences remain. In particular, the experimental signal of Fig. 4 exhibits an asymmetry between $\tau<0$ and $\tau>0$ that the model does not reproduce. One may argue that the use of a classical model to treat light-matter interactions is not accurate enough compared to quantummechanical models. The present description of nickel is crude and not nearly as sophisticated as those including the band structure in the Brillouin zone. ${ }^{45}$ One may also mention the theoretical studies devoted to the coherent photo-excitation of (Ga,Mn)As ferromagnetic semiconductors using linearly polarized light where the interplay between the optical nonlinearities, the spin-orbit interaction and the resulting optically induced magnetic anisotropy were treated microscopically. ${ }^{47-49}$ Let us also underline that we have worked with only one mode $\omega$ of the electromagnetic field without considering the spectral distribution $\Delta \omega$ of the laser pulse. Similarly, our description of the pump-probe configuration is clearly less developed than the reference work of Ref. 46. Also, we have not made any comments regarding the different contributions of "populations," "pump-polarization coupling," and "pump-perturbed free induction decay" usually present in a pump-probe description. ${ }^{23}$ Finally, the incorporation of the incoherent magnetization dynamic based on the stochastic Landau-Lifschitz-Gilbert equation would allow us to make comparisons between coherent and incoherent processes in a more detailed manner. We hope to tackle these issues in the near future.

However, we strongly believe that the conclusions of the present work already give meaningful insight into the physical mechanisms underlying the observed coherent magneto-optical phenomena. Hence, we propose to make some additional comments.

First, within the present semi-classical framework, the light-induced term $B_{\text {mat }}\left(1+\lambda\left(E_{y}-E_{x}\right)\right)$ included in the Lorentz force is crucial to reproduce the experimental trends. The latter are not recovered when $\lambda=0$, and it underlines the key role played by the directions of the electric field vectors in coherent magneto-optical phenomena. Then, according to the theoretical results depicted in Fig. 3, one may propose that the coherent effects (due to the nonlinear charge anisotropy and the light-induced molecular field) represent approximately $10 \%$ of the total ultrafast magnetic processes (for the particular experiment presented in Ref. 9). The remaining $90 \%$ may be attributed to the thermal effects. Then, under the assumptions of the present work, one may conclude that the coherent magnetic anisotropy measured through the experimental Kerr rotation angle is mostly due to the nonlinear modification of the orbital momenta driven by the Lorenz magnetic force. If one supposes that the spin dynamics is hidden in the light-induced coherent molecular mean-field its contribution consists in decreasing or increasing the value of the Lorenz force felt by the charge carriers. By roughly comparing the light-induced molecular mean-field to the magnetization dynamics the inset of Fig. 3 suggests that the magnetization change represents $\pm 25 \%$ of the value of the anisotropy produced by the charges. Concerning the microscopic origin of the light-induced molecular mean-field, the present study reveals the importance of the polarization state of the laser pulses on the modification of the magnetic state. This crucial physical ingredient may be incorporated in the model through a SOC involving the characteristics of the electric fields associated with the laser pulses. Finally, the interpretation proposed in Ref. 9 evoking the relativistic spin-photon interactions remains plausible.

Within a nonlinear and semi-classical Drude-Voigt model, we have investigated coherent ultrafast magnetooptical effects observed in time-resolved pump-probe experiments performed on ferromagnetic films. In order to explain the experimental results, a light-induced coherent molecular mean-field has been introduced whose microscopic origin originates from a spin-orbit-coupling involving the electron spins and the laser pulse electric 
field. The model successfully reproduces the observed experimental trends and gives meaningful insights into magneto-optical rotation behavior in the ultrafast regime. Theoretical predictions on further experimental studies are also proposed.

\section{ACKNOWLEDGMENTS}

Y.H. would like to thank H. Crespo and D. Schmool for their helpful comments and advices. Y.H. and P.-A.H. are also grateful to J.-Y. Bigot and M. Vomir for useful discussions. This work was supported in part by the Project No. NORTE-070124-FEDER-000070-Nanomateriais Multifuncionais and by the project ATOMAG supported by the European Research Council advanced Grant No. ERC2009-AdG-20090325-247452.

\section{APPENDIX: PRECISIONS ON THE CLASSICAL PUMP- PROBE TERM}

We give some details regarding the obtention of Eq. (16). The vector displacement due to the pump excitation reads $\mathbf{r}_{b}^{(1), \text { pump }}(\Omega) \equiv \mathbf{r}_{b 0}^{\text {pump }}(\Omega) G(t) e^{i \Omega t}$ with $G(t)=e^{-\frac{t^{2}}{2 \Gamma^{2}}}$. Similarly, the spatial displacement induced by the probe beam, $\mathbf{r}^{\text {probe }}(\omega)$, is expressed as $\mathbf{r}_{b}^{\text {probe }}(\omega) \equiv \mathbf{r}_{b 0}^{\text {probe }}(\omega) g(t, \tau) e^{i \omega(t-\tau)}$ with $g(t, \tau)=e^{-\frac{(t-\tau)^{2}}{2 \gamma^{2}}}$. Both terms contribute to the nonlinear force $b\left(\mathbf{r}_{b}^{(1)}\right)^{3}$, which reads as follows:

$$
\begin{aligned}
& \left(\mathbf{r}_{b}^{(1)}\right)^{3}=\left[\mathbf{r}_{b}^{(1), \text { pump }}(\Omega)+\mathbf{r}_{b}^{(1), \text { pump }}(-\Omega)+\mathbf{r}_{b}^{(1), \text { prope }}(\omega)+\mathbf{r}_{b}^{(1), \text { probe }}(-\omega)\right]^{3} \\
& =\left[\mathbf{r}_{0 b}^{\mathrm{pump}}(\Omega) G(t) e^{i \Omega t}+\mathbf{r}_{0 b}^{\mathrm{pump}}(-\Omega) G(t) e^{-i \Omega t}+\mathbf{r}_{0 b}^{\mathrm{probe}}(\omega) g(t, \tau) e^{i \omega(t-\tau)}+\mathbf{r}_{0 b}^{\mathrm{probe}}(-\omega) g(t, \tau) e^{-i \omega(t-\tau)}\right]^{3} \\
& =[\underbrace{\left(\mathbf{r}_{0 b}^{\mathrm{pump}}(\Omega) G(t) e^{i \Omega t}+\mathbf{r}_{0 b}^{\mathrm{pump}}(-\Omega) G(t) e^{-i \Omega t}\right)^{3}}_{\mathrm{A}: 3 \Omega,-3 \Omega, \Omega,-\Omega}+\underbrace{\left(\mathbf{r}_{0 b}^{\mathrm{probe}}(\omega) g(t, \tau) e^{i \omega(t-\tau)}+\mathbf{r}_{0 b}^{\mathrm{probe}}(-\omega) g(t, \tau) e^{-i \omega(t-\tau)}\right)^{3}}_{\mathrm{B}: 3 \omega,-3 \omega, \omega,-\omega} \\
& +3 \underbrace{\left(\mathbf{r}_{0 b}^{\mathrm{pump}}(\Omega) G(t) e^{i \Omega t}+\mathbf{r}_{0}^{\mathrm{pump}}(-\Omega) G(t) e^{-i \Omega t}\right)^{2}\left(\mathbf{r}_{0 b}^{\mathrm{probe}}(\omega) g(t, \tau) e^{i \omega(t-\tau)}+\mathbf{r}_{0 b}^{\mathrm{probe}}(-\omega) g(t, \tau) e^{-i \omega(t-\tau)}\right)}_{\mathrm{C}:(2 \Omega+\omega),(2 \Omega-\omega),(-2 \Omega+\omega),(-2 \Omega-\omega), \omega,-\omega} \\
& +3 \underbrace{\left(\mathbf{r}_{0}^{\mathrm{pump}}(\Omega) G(t) e^{i \Omega t}+\mathbf{r}_{0 b}^{\mathrm{pump}}(-\Omega) G(t) e^{-i \Omega t}\right)\left(\mathbf{r}_{0 b}^{\mathrm{probe}}(\omega) g(t, \tau) e^{i \omega(t-\tau)}+\mathbf{r}_{0 b}^{\mathrm{probe}}(-\omega) g(t, \tau) e^{-i \omega(t-\tau)}\right)^{2}}_{\mathrm{D}:(2 \omega+\Omega),(2 \omega-\Omega),(-2 \omega+\Omega),(-2 \omega-\Omega), \Omega,-\Omega}] .
\end{aligned}
$$

The previous expression is composed of 4 terms (A, B, C, and D) which may be expanded into 24 terms vibrating at different frequencies. We indicate below the brackets, the different Fourier components which may be obtained for each term. Because we are focusing on the $\omega$ mode representing the contribution of the probe pulse, one needs to keep only the terms vibrating at $\omega$ which are present in B and C. It results in the expression given in Eq. (16)

$$
\begin{aligned}
\left(\mathbf{r}_{b}^{(1)}\right)^{3} \approx & 3 \times\left(\mathbf{r}_{0 b}^{\text {probe }}(\omega) g(t, \tau) e^{i \omega(t-\tau)}\right)^{2} \times \mathbf{r}_{0 b}^{\text {probe }}(-\omega) g(t, \tau) e^{-i \omega(t-\tau)} \\
& +3 \times 2 \times\left(\mathbf{r}_{0 b}^{\text {pump }}(\Omega) G(t) e^{i \Omega t}\right) \times\left(\mathbf{r}_{0 b}^{\text {pump }}(-\Omega) G(t) e^{-i \Omega t}\right) \times\left(\mathbf{r}_{0 b}^{\text {probe }}(\omega) g(t, \tau) e^{i \omega(t-\tau)}\right) \\
\approx & 3\left(\mathbf{r}_{b}^{(1), \text { probe }}(\omega) \mathbf{r}_{b}^{(1) \text { probe }}(\omega)\right) \mathbf{r}_{b}^{(1), \text { probe }}(-\omega)+6\left(\mathbf{r}_{b}^{(1), p u m p}(\Omega) \mathbf{r}_{b}^{(1), \text { pump }}(-\Omega)\right) \mathbf{r}_{b}^{(1), \text { probe }}(\omega) .
\end{aligned}
$$

${ }^{1}$ J.-Y. Bigot, C. R. Acad. Sci., Ser. IV: Phys. 2, 1483 (2001).

${ }^{2}$ A. Kirilyuk, A. V. Kimel, and Th. Rasing, Rev. Mod. Phys. 82, 2731 (2010).

${ }^{3}$ J.-Y. Bigot and M. Vomir, Ann. Phys. (Berlin) 525, 2 (2013).

${ }^{4}$ P. M. Oppeneer and A. Liebsch, J. Phys.: Condens. Matter 16, 5519 (2004).

${ }^{5}$ O. Chubykalo-Fesenko, U. Nowak, R. W. Chantrell, and D. Garanin, Phys. Rev. B 74, 094436 (2006).

${ }^{6}$ M. Lisowski, P. A. Loukakos, A. Melnikov, I. Radu, L. Ungureanu, M. Wolf, and U. Bovensiepen, Phys. Rev. Lett. 95, 137402 (2005).

${ }^{7}$ G. Lefkidis, G. P. Zhang, and W. Hübner, Phys. Rev. Lett. 103, 217401 (2009).

${ }^{8}$ G. P. Zhang, W. Hübner, G. Lefkidis, Y. Bai, and T. F. George, Nat. Phys. 5, 499 (2009)

${ }^{9}$ J.-Y. Bigot, M. Vomir, and E. Beaurepaire, Nat. Phys. 5, 515 (2009).

${ }^{10}$ B. Koopmans, G. Malinowski, F. Dalla Longa, D. Steiauf, M. Fähnle, T. Roth, M. Cinchetti, and M. Aeschlimann, Nat. Mater. 9, 259 (2010).

${ }^{11}$ A. Eschenlohr, M. Battiato, P. Maldonado, N. Pontius, T. Kachel, K. Holldack, R. Mitzner, A. Fhlisch, P. M. Oppeneer, and C. Stamm, Nat. Mater. 12, 332 (2013).
${ }^{12}$ O. Morandi, P. A. Hervieux, and G. Manfredi, New J. Phys. 11, 073010 (2009).

${ }^{13}$ O. Morandi, P.-A. Hervieux, and G. Manfredi, Eur. Phys. J. D 52, 155 (2009).

${ }^{14}$ O. Morandi, P.-A. Hervieux, and G. Manfredi, Phys. Rev. B 81, 155309 (2010).

${ }^{15}$ C. Boeglin, E. Beaurepaire, V. Halté, V. Lopez-Flores, C. Stamm, N. Pontius, H. Dürr, and J.-Y. Bigot, Nature 465, 458 (2010).

${ }^{16} \mathrm{G}$. Lefkidis and W. Hübner, Phys. Rev. B 87, 014432 (2013).

${ }^{17}$ Y. Hinschberger and P.-A. Hervieux, Phys. Rev. B 88, 134413 (2013).

${ }^{18}$ E. Beaurepaire, J. C. Merle, A. Daunois, and J.-Y. Bigot, Phys. Rev. Lett. 76, 4250 (1996).

${ }^{19}$ R. F. L. Evans, W. J. Fan, P. Chureemart, T. A. Ostler, M. O. A. Ellis, and R. W. Chantrell, J. Phys.: Condens. Matter 26, 103202 (2014).

${ }^{20}$ H. R. Hulme, Proc. R. Soc. London, Ser. A 135, 237 (1932).

${ }^{21}$ P. N. Argyres, Phys. Rev. 97, 334 (1955).

${ }^{22}$ H. S. Bennett and E. A. Stern, Phys. Rev. 137, A448 (1965).

${ }^{23}$ H. Vonesh and J.-Y. Bigot, Phys. Rev. B 85, $180407(\mathrm{R})$ (2012).

${ }^{24}$ Y. Hinschberger and P.-A. Hervieux, Phys. Lett. A 376, 813 (2012).

${ }^{25}$ Y. Hinschberger and P.-A. Hervieux, Phys. Lett. A 379, 2261 (2015). 
${ }^{26}$ A. Dixit, Y. Hinschberger, G. Manfredi, and P.-A. Hervieux, Phys. Rev. A 88, 032117 (2013).

${ }^{27}$ Y. Hinschberger, A. Dixit, G. Manfredi, and P.-A. Hervieux, Phys. Rev. A 91, 012101 (2015).

${ }^{28}$ R. Mondal, M. Berritta, K. Carva, and P. M. Oppeneer, Phys. Rev. B 91, 174415 (2015).

${ }^{29}$ M. Barthelemy, M. Vomir, M. Sanches Piaia, H. Vonesch, P. Molho, B. Barbara, and J.-Y. Bigot, e-print arXiv:1311.0080v1.

${ }^{30}$ C. S. Gonalves, A. S. Silva, D. Navas, M. Miranda, F. Silva, H. Crespo, and D. S. Schmool, in Ultrafast Magnetism I, edited by J. Y. Bigot, W. Hubner, T. Rasing, and R. Chantrell (Springer, Strasbourg, France, 2015), Vol. 159, pp. 53-55.

${ }^{31}$ Y. Hinschberger and J.-P. Lavoine, J. Appl. Phys. 118, 053908 (2015).

${ }^{32}$ Z. Q. Qiu and S. D. Bader, Rev. Sci. Instrum. 71, 1243 (2000).

${ }^{33}$ A. D. Slepkov, F. A. Hegmann, Y. Zhao, R. R. Tykwinski, and K. Kamada, J. Chem. Phys. 116, 3834 (2002).

${ }^{34}$ C. Zener, Phys. Rev. 83, 299 (1951).

${ }^{35}$ C. Kittel, Introduction to Solid State Physics (John Wiley and Sons, New York, 1996)

${ }^{36}$ K. M. Dani, J. Tignon, M. Breit, D. S. Chemla, E. G. Kavousanaki, and I. E. Perakis, Phys. Rev. Lett. 97, 057401 (2006).
${ }^{37}$ S. Schmitt-Rink et al., Phys. Rev. B 32, 6601 (1985).

${ }^{38}$ D. Wang and M. M. Dignam, Phys. Rev. B 79, 165320 (2009).

${ }^{39}$ R. W. Boyd, Nonlinear Optics (Academic Press, 2003).

${ }^{40}$ Handbook of Optical Constants of Solids, edited by E. D. Palik (Academic Press, Orlando, 1985).

${ }^{41}$ M. A. Ordal, L. L. Long, R. J. Bell, S. E. Bell, R. R. Bell, R. W. Alexander, Jr., and C. A. Ward, Appl. Opt. 22, 1099 (1983).

${ }^{42}$ S. N. Paul, S. K. Bhattacharya, S. Mondal, and B. Chakraborti, Fiz. A 13, 161 (2004).

${ }^{43}$ L. B. Scaffardi and J. Tocho, Nanotechnology 17, 1309 (2006).

${ }^{44}$ B. Koopmans, M. van Kampen, and W. J. M. de Jonge, J. Phys.: Condens. Matter 15, S723-S736 (2003).

${ }^{45}$ G. P. Zhang, Y. Bai, W. Hübner, G. Lefkidis, and T. F. George, J. Appl. Phys. 103, 07B113 (2008).

${ }^{46}$ H. Brito Cruz, J. P. Gordon, P. C. Becker, R. L. Fork, and C. V. Shank, Quantum Electron. 24, 261 (1988).

${ }^{47}$ J. Chovan and I. E. Perakis, Phys. Rev. B 77, 085321 (2008).

${ }^{48}$ M. D. Kapetanakis, I. E. Perakis, K. J. Wickey, C. Piermarocchi, and J. Wang, Phys. Rev. Lett. 103, 047404 (2009).

${ }^{49}$ M. D. Kapetanakis, P. C. Lingos, C. Piermarocchi, J. Wang, and I. E. Perakis, Appl. Phys. Lett. 99, 091111 (2011). 